\title{
MedienPädagogik
}

Zeitschrift für Theorie und Praxis der Medienbildung

Themenheft Nr. 41: Inklusiv-mediale Bildung in schulischen Kontexten. Eine interdisziplinäre Bestandsaufnahme

Herausgegeben von Christian Filk und Heike Schaumburg

\section{Personalisiertes Lernen mit digitalen Medien als Herausforderung für die Schulentwicklung}

\author{
Ein systematischer Forschungsüberblick
}

Heike Schaumburg

\begin{abstract}
Zusammenfassung
Ein zentraler Mehrwert digitaler Medien im inklusiven Unterricht ist die technische Unterstützung der Individualisierung von Lernprozessen. Unter dem Stichwort des «digital gestützten personalisierten Lernens» wird diese seit etwa einem Jahrzehnt von vielen Schulen weltweit erprobt. Mittlerweile liegen zahlreiche Forschungsarbeiten vor, die in der Debatte um Inklusion und Digitalisierung bislang weitgehend ignoriert werden. Das Anliegen des vorliegenden Textes ist es deshalb, den Stand der Forschung zum digital gestützten personalisierten Lernen aufzuarbeiten, um daraus Erkenntnisse abzuleiten, die auch für die Weiterentwicklung inklusiv-medialer Schul- und Unterrichtskonzepte relevant sind. Der nach den Vorgaben des ENTREQ-Statements erstellte systematische Forschungsbericht fasst die Ergebnisse von 18 Studien zusammen, in denen die Erfahrungen zahlreicher Pilotversuche ausgewertet wurden. Im Mittelpunkt steht dabei die Frage, wie sich die Einführung von digital gestütztem personalisiertem Lernen auf die Schulentwicklung auswirkt. Unter Verwendung des Drei-Wege-Modells der Schulentwicklung (Rolff und Thünken 2020; Rolff 1998) fasst der Überblick die Ergebnisse auf Unterrichts-, Personal- und Organisationsebene zusammen. Die Ergebnisse zeigen ein breites Spektrum von Umsetzungen. Ein bedeutender Mehrwert des digital gestützten personalisierten Lernens wird in der Bereitstellung zusätzlicher lernprozessbezogener Daten gesehen. Zahlreiche Barrieren auf allen drei Ebenen der Schulentwicklung behindern eine umfassende Implementierung.
\end{abstract}

Personalized Learning with Digital Media as a Challenge for School Development. A Systematic Research Review

\begin{abstract}
A major benefit of digital media in inclusive education is the technical support of individualized learning processes. «Technology-enhanced personalized learning» is a term that has been used for about a decade to describe this kind of systematic individualization with the help of digital learning platforms and programs. In the meantime, numerous
\end{abstract}


research studies have been published that have been largely ignored in the debate about inclusion and digitization. The purpose of this paper is therefore to review the current state of research on personalized learning in order to derive insights that are also relevant for the further development of inclusive digital school and teaching concepts. This systematic research review, which follows the rules of the ENTREQ-Statement, summarizes the results of 18 studies evaluating numerous pilot experiments. It focuses on the effects of the introduction of technology-enhanced personalized learning on school development. Using the three-way model of school development (Rolff und Thünken 2020; Rolff 1998), the review summarizes findings at the instructional, personnel, and organizational level. The results show a wide range of implementations. A significant added value of technology-enhanced personalized learning is seen in the provision of additional student data. Numerous barriers at all three levels of school development hinder an extensive implementation.

\section{Inklusion, Digitalisierung und personalisiertes Lernen}

Die Einbindung digitaler Medien im inklusiven Unterricht verfolgt unterschiedliche Zielsetzungen. Neben der Vermittlung medienbezogener Kompetenzen als Voraussetzung von Partizipation in einer digitalen Netzwerkgesellschaft (Filk 2019) und der Ermöglichung von schulischer Teilhabe durch assistive Technologien (Bosse 2019) ist die individuelle Lernförderung von Schülerinnen und Schülern mit heterogenen Lernvoraussetzungen ein häufig vorgebrachtes Argument dafür, den Einsatz digitaler Medien im inklusiven Unterricht zu verstärken. Dabei besteht in der Einschätzung, dass digitale Medien ein bedeutsames Potenzial für die Individualisierung des Lernens bergen, ebenso grosse Einigkeit, wie in der Kritik am Fehlen von entsprechender Forschung, die die vermuteten Potenziale belegen könnte (Bosse 2019, Schulz et al. 2019; s. auch Hartung et al. 2021 sowie Schulz 2021 in diesem Themenheft).

Der vorliegende Beitrag setzt an diesem Forschungsdesiderat an. Denn wenngleich der Einsatz digitaler Medien zur individualisierten Lernförderung im inklusiven Unterricht im deutschsprachigen Raum bislang noch selten untersucht wurde, so ist in der anglo-amerikanischen Literatur in den letzten Jahren eine rege Forschungstätigkeit zu den Potenzialen digitaler Medien insbesondere für die individualisierte Lernförderung in heterogenen Lerngruppen zu verzeichnen. Allerdings findet sich diese unter dem im deutschsprachigen Raum noch kaum verbreiteten Begriff «technology-enhanced personalized learning» (Holmes et al. 2018). Seit der Jahrtausendwende hat dieses Konzept weltweit Eingang in bildungspolitische Initiativen und Programme gefunden (Duckett 2010). Insbesondere in den USA wurden im letzten Jahrzehnt im Rahmen gross angelegter nationaler Bildungsinitiativen erhebliche Summen in die Förderung der computergestützten Individualisierung und adaptiven Gestaltung von Lernprozessen investiert. Infolgedessen erlebte die technische 
Entwicklung von Lernplattformen und -programmen einen massiven Schub, mit dem sowohl die Entwicklung von auf künstlicher Intelligenz basierenden Lern- und Übungsprogrammen vorangetrieben wurde, als auch die Technologie des «Educational Data Mining» weiterentwickelt wurde, also die Personalisierung digitaler Lernangebote auf der Grundlage der systematischen Analyse der immensen Datenmengen, die bei der Nutzung von Lernplattformen anfallen. Nicht zuletzt begaben sich zahlreiche Schulen auf den Weg, ihre Lerninfrastrukturen grundsätzlich umzugestalten, um sich dem Idealbild einer Lernumgebung anzunähern, in der die Schülerinnen und Schüler konsequent entsprechend ihrer individuellen Lernbedürfnisse unterstützt werden (Enyedy 2014).

Dieser Text beschäftigt sich schwerpunktmässig mit der Frage, was die Einführung solcher digitalen Systeme, die eine umfassende Individualisierung von Lernprozessen erlauben, für die Einzelschule als Organisation bedeutet. Welche Erfahrungen wurden mit der Einführung solcher Systeme gemacht? Welche Vorteile und Potenziale bestätigen sich in der Praxis? Welche Hindernisse und Herausforderungen stellen sich und wie können sie überwunden werden? Und was bedeutet dies für inklusivdigitale Schul- und Unterrichtsentwicklung?

Im ersten Teil des Beitrags wird zunächst das Konstrukt «digital gestütztes personalisiertes Lernen» anhand seiner zentralen didaktischen Dimensionen in seiner Vielschichtigkeit charakterisiert, um seine Anschlussfähigkeit an den Diskurs um Inklusion und Digitalisierung aufzuzeigen. Nachfolgend werden Überlegungen dazu angestellt, was die Umsetzung personalisierten Lernens als Aufgabe für die Schulentwicklung bedeutet und inwiefern Forschungsbefunde zum personalisierten Lernen auch für eine inklusiv-mediale Schulentwicklung interessant sein könnten. Im zweiten Teil schliesst sich ein Systematic Review des anglo-amerikanischen Forschungsstands nach der Methodik des ENTREQ-Statements an. Im Mittelpunkt steht dabei die Frage, welche Herausforderungen sich bei der Implementierung personalisierten Lernens für die Schulentwicklung stellen. Die im Rahmen zahlreicher Evaluationen von Pilotprojekten und Modellversuchen gesammelten Erkenntnisse werden dabei entlang der Dimensionen des Drei-Wege-Modells der Schulentwicklung nach Rolff und Thünken (2020) geordnet und systematisiert. Abschliessend wird ein Versuch unternommen, die Vielfalt der Befunde zusammenzufassen und Schlussfolgerungen für eine inklusiv-medialer Schulentwicklung abzuleiten.

\section{Was ist (digital gestütztes) personalisiertes Lernen?}

Die Unschärfe und Vielschichtigkeit des Konstrukts «personalisiertes Lernen» wird allseits beklagt (Enyedy 2014). Grob gesagt wird als «personalised learning» im anglo-amerikanischen Sprachraum ein didaktisches Konzept bezeichnet, bei dem Lernsituationen und -materialien so gestaltet werden, dass sie den individuellen (und 
heterogenen) Lernvoraussetzungen und -bedürfnissen einzelner Schülerinnen und Schüler bestmöglich Rechnung tragen (Graf und Kinshuk 2012). Schaumburg (2021) arbeitet heraus, dass sich hinter dieser recht allgemeinen Definition vielfältige Gestaltungsoptionen für personalisiertes Lernen verbergen, die sich entlang dreier Dimensionen systematisieren lassen:

1. Didaktische Entscheidungsfelder (Personalisierung von Lernzielen, Lerninhalten, Lernmethoden, Lernpfaden, Lernzeit, Lernort),

2. Eigenschaften der Schülerinnen und Schüler (Personalisierung mit Blick auf Vorwissen, Leistungsfähigkeit, Interesse, Motivation, Lernstil) sowie

3. Locus of Control (Steuerung und Auswahl des personalisierten Lernmaterials extern, d. h. durch die Lehrkraft bzw. durch ein Computerprogramm oder intern, d. h. durch die Schülerinnen und Schüler).

Diese Dimensionen bilden ein Raster anhand dessen leicht nachvollzogen werden kann, dass sich Lernprozesse mit digitalen Medien, je nach Typ des eingesetzten Programms und der Art und Weise seiner Nutzung, in ganz unterschiedlicher Form personalisieren lassen:

So ermöglicht ein digitales Übungsprogramm wie etwa die web-basierte «AntonApp» in didaktischer Hinsicht in erster Linie eine Personalisierung mit Blick auf Lernzeit und Lernpfad. Sofern Lernaufgaben von den Lehrkräften individuell zugeordnet werden und die Schülerinnen und Schüler auch ausserhalb des Unterrichts mit der App arbeiten, können darüber hinaus Lernziel, Lerninhalt und Lernort personalisiert werden. Die Anton-App spricht hinsichtlich der Eigenschaften der Lernenden vor allem unterschiedliche Wissensstände und Leistungsniveaus an. Der Locus of Control liegt bei der Lehrkraft bzw. bei dem Programm, das den Schülerinnen und Schülern individuell auf ihr Niveau zugeschnittene Aufgaben vorlegt.

Ein anderes Beispiel wäre die Nutzung des Internets im Rahmen einer komplexen Projektaufgabe, bei der innerhalb eines bestimmten Rahmens Informationen zu einem selbst gewählten Thema recherchiert werden sollen. In didaktischer Hinsicht erlaubt der Einsatz digitaler Medien hier eine weitreichende Personalisierung (Lernzeit, Lernpfad, Lernmethode, Lerninhalt, Lernziel, Lernort). Dabei können neben verschiedenen Wissensständen auch Interessen und Motivation der Schülerinnen und Schüler einbezogen werden. Der Locus of Control liegt in diesem Fall eher bei den Schülerinnen und Schülern, die weitreichende Entscheidungen bezüglich ihres Lernprozesses selbst zu treffen haben.

Mediendidaktisch betrachtet, verbergen sich hinter dem Begriff «Personalisierung» zwei widersprüchliche Grundauffassungen: Der Begriff bezeichnet einerseits die datengestützte Diagnose, Strukturierung und Optimierung von Lernprozessen im Geiste der behavioristisch-kognitivistischen Tradition des US-amerikanischen Instruktionsdesigns (Dockterman 2018). Andere Autoren vertreten dagegen einen 
diametral entgegengesetzten, eher konstruktivistischen Ansatz, in dessen Zentrum die Selbststeuerung, Autonomie und Verantwortung der Lernenden für die Gestaltung ihrer je individuellen Lernprozesse stehen (Kallick und Zmuda 2017; Schratz und Westfall-Greiter 2010, s. auch Schaumburg 2021).

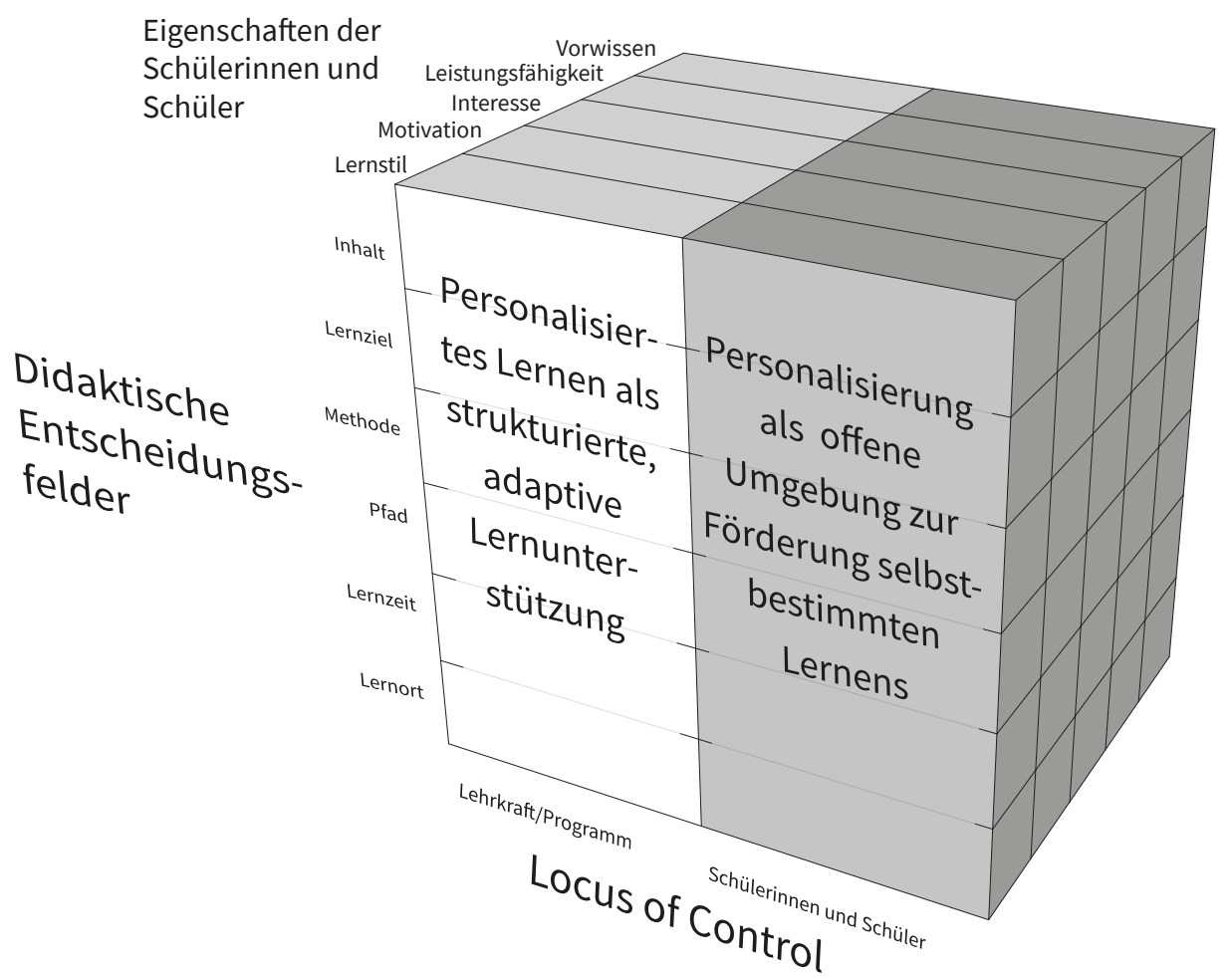

Abb. 1.: Dimensionen und Grundauffassungen personalisierten Lernens (Schaumburg 2021).

Mit Blick auf den inklusiven Unterricht sind jedoch beide Perspektiven anschlussfähig: Die erste, kognitivistisch-instruktionale Sichtweise weist eine deutliche Nähe zu Konzepten des adaptiven Unterrichts auf, der auch in inklusiven Settings als zielführend erachtet wird. So betonen Schulz et al. (2019) sowie Bosse (2019) den Mehrwert adaptiver Lernprogramme im inklusiven Unterricht, da sie einen Beitrag dazu leisten, das geforderte hochgradig differenzierte Lernangebot bereitzustellen und den Lehrkräften zusätzliche Ressourcen für die individuelle Diagnostik zu bieten.

Die eher konstruktivistische Auffassung schliesst dagegen an ein Inklusionsverständnis an, dass in der Partizipation und Mitbestimmung aller Schülerinnen und Schüler (Boban und Hinz 2009) und dem Lernen an einem gemeinsamen Gegenstand (Feuser 2013) wichtige Zielperspektiven des inklusiven Unterrichts sieht. Vor diesem Hintergrund haben auch und gerade offene digitale Lernumgebungen, die gemeinsames selbstbestimmtes und exploratives Lernen erlauben, ihren Platz im inklusiven Unterricht (Schaumburg 2020). 


\section{Personalisiertes Lernen mit digitalen Medien als Schulentwicklungsaufgabe}

Auch wenn Lehrkräfte grundsätzlich mit der Einbindung binnendifferenzierter Aufgabenstellungen und Lernangebote im Unterricht vertraut sind, eröffnet die Implementation digitaler personalisierter Lernumgebungen weitreichende Perspektiven für die Veränderung von Unterricht. Die erweiterten Möglichkeiten der Individualisierung des Lernens können, wie oben ausgeführt, sehr unterschiedliche Formen annehmen. In der Unterrichtsentwicklung müssen diese Möglichkeiten durchdacht und für die jeweilige Lerngruppe angepasst und nutzbar gemacht werden. So müssen im Unterricht zeitliche und organisatorische Strukturen für personalisiertes Lernen geschaffen, inhaltliche und methodische Entscheidungen getroffen, Unterstützungsangebote und Rückmeldungsformate konzipiert werden usw. Ggf. bringt die Einführung personalisierten Lernens tiefgreifende Veränderungen der Unterrichtskultur und der Lehrkräfterolle mit sich (Kallick und Zmuda 2017). Ganz ähnliche Herausforderungen werden auch für die Einführung digitaler Medien diskutiert. Auch diese brechen gewohnte Unterrichtsroutinen auf und erfordern eine Neuausrichtung von Unterricht und schulischem Lernen (Schaumburg und Prasse 2019, vgl. auch Hartung et al. 2021 in diesem Themenheft).

Personalisiertes Lernen mit digitalen Medien ist dabei kaum sinnvoll von einzelnen Lehrkräften in individuellen Klassen implementierbar, sondern weist in der Regel über den einzelnen Klassenraum hinaus. Es besteht deshalb Einigkeit, dass es als umfassende Schulentwicklungsaufgabe gedacht werden sollte (Bingham et al. 2018; Holmes et al. 2018). Aufgaben, die sich bei der Implementierung digital gestützten personalisierten Lernens auf der Ebene der Einzelschule stellen, betreffen z. B. die Entwicklung eines schulinternen Curriculums, die zeitliche Strukturierung des Schultags, um Freiräume für individualisiertes Lernen zu schaffen, die Entwicklung der IT-Infrastruktur der Schule, die Fortbildung von Lehrkräften oder die Stärkung der Kooperation im Kollegium (Holmes et al. 2018; de Hond und Rood 2017). Personalisierung des Lernens ist somit nicht allein eine Frage der Unterrichtsentwicklung, sondern berührt in gleicher Weise die Personal- und Organisationsentwicklung einer Schule. Zur Modellierung der Schulentwicklungsprozesse, die mit der Einführung digital gestützten personalisierten Lernens verbunden sind, eignet sich deshalb das Drei-Wege-Modell der Schulentwicklung von Rolff (1998), das genau diese drei Felder auffächert. Das Modell wurde bereits von verschiedenen Autorinnen und Autoren (Schulz-Zander 1999; Zylka 2018) für die Medienentwicklung an Schulen adaptiert, indem den drei ursprünglichen Entwicklungsfeldern Unterrichts-, Personal- und Organisationsentwicklung ein weiteres, technologie- bzw. digitalisierungsbezogenes hinzugefügt wurde. Der vorliegende Beitrag folgt jedoch der Weiterentwicklung von Rolff und Thünken (2020), die die Digitalisierung nicht als weiteres Feld sehen, sondern digital gestütztes Lernen im Zentrum des Schulentwicklungsprozesses ansiedeln, auf das sich die drei Entwicklungsfelder Unterrichts-, Personal- und 
Organisationsentwicklung als miteinander verschränkte Prozesse beziehen. Im Rahmen des vorliegenden Beitrags wird dieses Modell für die Betrachtung des digital gestützten personalisierten Lernens adaptiert. Im Modell von Rolff und Thünken (2020) ist die Personalisierung des Lernens bereits enthalten, sie wird jedoch primär als eine Aufgabe der Unterrichtsentwicklung modelliert. Davon abweichend wird digital gestütztes personalisiertes Lernen im Rahmen des vorliegenden Beitrags ins Zentrum des Modells gerückt, in der Annahme, dass es, wie oben angerissen, alle drei Dimensionen der Schulentwicklung berührt (vgl. Abb. 2).

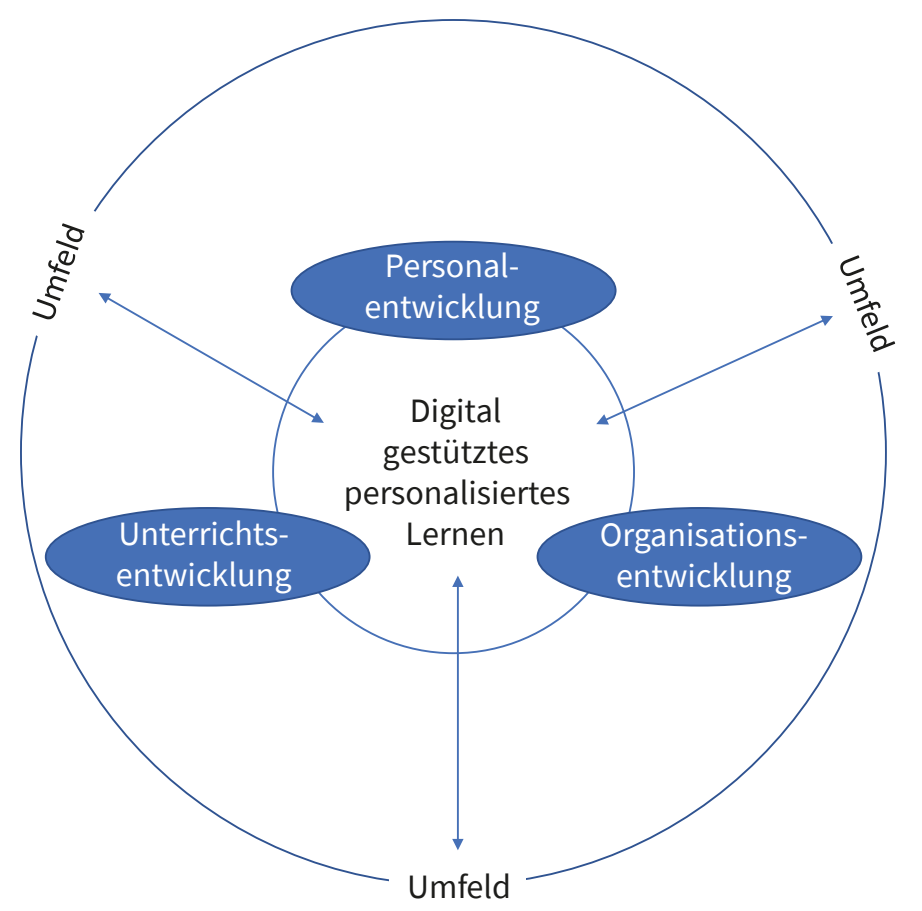

Abb. 2.: Drei-Wege-Modell der Schulentwicklung adaptiert nach Rolff und Thünken (2020).

Für den im Folgenden dargestellten Systematic Review wird das Modell als Folie genutzt, um die vorliegenden Forschungsergebnisse zu den Anforderungen an die Schulentwicklung bei der Implementierung digital gestützten personalisierten Lernens zu systematisieren. Im Vordergrund stehen dabei folgende Fragen:

1. Wie wird digital gestütztes personalisiertes Lernen implementiert? Welches lerntheoretisch-didaktische Verständnis (s. o.) von personalisiertem Lernen legen Schulen ihrem Entwicklungsprozess zugrunde? Wo liegt ihr Fokus bei der Einführung personalisierten Lernens? Welche Strategien oder Modelle für den Einsatz personalisierten Lernens lassen sich unterscheiden? 
2. Welche Barrieren oder Hindernisse beinträchtigen die Einführung von personalisiertem Lernen in den drei Bereichen Unterrichts-, Personal- und Organisationsentwicklung?

3. Welche Lösungsansätze und Gelingensbedingungen zeichnen sich in den drei Entwicklungsfeldern ab?

4. Lassen sich übergeordneten Erkenntnisse für die inklusiv-mediale Schulentwicklung aus den Ergebnissen ableiten?

\section{Methode}

Dieser Review folgt in seinem Vorgehen dem ENTREQ-Statement (Tong et al. 2012). Es handelt sich hierbei um ein Verfahren zur Erhöhung der Transparenz bei der Zusammenfassung des Forschungsstands qualitativer Studien, das den Rechercheprozess und seine Ergebnisse in die fünf Teilbereiche a) Forschungsfragen, b) Methode, c) Literatursuche und Auswahl, d) Bewertung sowie e) Synthese der Ergebnisse gliedert, die differenziert dargestellt und offengelegt werden sollen. Für jeden Teilbereich werden jeweils nochmals Einzelschritte zum Vorgehen spezifiziert, so dass der Gesamtprozess aus 21 Vorgehensindikatoren besteht, die im Folgenden abgearbeitet werden.

Ausgehend von den im vorigen Abschnitt formulierten Forschungsfragen orientiert sich dieser Review an der Methode der thematischen Zusammenfassung (Thomas und Harden 2008). Wie bereits erläutert, bildet das adaptierte Drei-WegeSchulentwicklungsmodell von Rolff und Thünken (2020) die theoretische Grundlage für die Analyse des Forschungsstands und die Systematisierung der Forschungsbefunde.

\subsection{Literatursuche}

Für die Literatursuche wurden die Forschungsdatenbanken ProQuest und die Literatursuche des Fachportals Pädagogik genutzt. Als Meta-Datenbank vereint ProQuest die Einträge von 47 Datenbanken und erlaubt Zugriff auf die Inhalte mehrerer tausend wissenschaftlicher Zeitschriften und Bücher sowie Qualifikationsschriften. Da ProQuest ausserdem auch eine Fülle nicht-wissenschaftlicher Quellen enthält und um die Qualität der Ergebnisse zu erhöhen, wurde die Suche eingeschränkt auf Publikationen, die einem Peer Review unterzogen wurden. Weiterhin wurde der Publikationszeitraum auf die Jahre 2010 bis 2020 begrenzt, da, wie eingangs erwähnt, in diesem Zeitraum gerade in technischer Hinsicht eine beträchtliche Weiterentwicklung stattgefunden hat. Suchbegriffe waren «personalized/personalised learning», «personalization/personalization», «school», «school development», «school organization/organization», «implementation». Mit diesen Begriffen wurden Titel, Schlagworte 
und Abstracts durchsucht. Mit der Begriffskombination «personalis/zed learning + school» sowie mit einer Titelsuche mit den Suchbegriffen «personalis $/ \mathrm{z}^{*}+$ school» wurden schliesslich 1135 potenziell relevante Einträge identifiziert. Die deutschsprachigen Suchbegriffe "personalisiertes Lernen», "Personalisierung», «Schule», «Schulentwicklung», «Schulorganisation» ergaben keine relevanten Treffer in der Datenbank ProQuest. Im Fachportal Pädagogik konnten mit den Suchbegriffen «personalisiertes Lernen» sowie «Personalisierung + Schule» 30 potenziell relevante Einträge, die seit 2010 veröffentlicht wurden, lokalisiert werden. Bei der Recherche im Fachportal Pädagogik wurde nicht berücksichtigt, ob die Texte einem Peer Review unterzogen wurden.

\subsection{Ein- und Ausschluss von Studien}

Die gefundenen 1.165 Einträge wurden im nächsten Schritt auf folgende Einschlusskriterien hin untersucht:

1. Der Text berichtet originäre empirische Ergebnisse, die in einem strukturierten, nachvollziehbar beschriebenen Forschungsprozess gewonnen wurden,

2. Die Untersuchung wurde an einer Primar- oder Sekundarschule durchgeführt, an der personalisiertes Lernen praktiziert wird oder wurde,

3. Die Untersuchung richtet sich schwerpunktmässig auf Prozesse der Schulentwicklung in mindestens einem der drei Felder Unterrichts-, Personal- oder Organisationsentwicklung.

Der Selektionsprozess erfolgte sukzessive im ersten Schritt auf der Grundlage der Titel der Arbeiten, anschliessend unter Einbezug der Abstracts und im letzten Schritt auf Basis der Lektüre des gesamten Textes. Ausgeschlossen wurden auf diese Weise programmatische Texte und Zusammenfassungen ohne eigenen empirischen Anteil sowie Erfahrungsberichte. Weiterhin fanden empirische Studien an ausserschulischen Institutionen, Institutionen der Lehrkräftebildung und Laborstudien keine Berücksichtigung. Ausgeschlossen wurden schliesslich auch Studien, die vorrangig die Lernwirksamkeit personalisierter Lernumgebungen untersuchen, aber keine verwertbaren Ergebnisse zur schulischen Implementation enthielten.

Auf diese Weise wurden 16 relevante Untersuchungen identifiziert. Durch Rückwärtssuchen in diesen wurden anschliessend zwei weitere, häufig referenzierte Studien ausfindig gemacht (Basham et al. 2016; Pane et al. 2017) und nachträglich in die Auswahl eingeschlossen. 


\subsection{Codierung}

Die eingeschlossenen 18 Studien wurden nachfolgend mit dem Literaturverwaltungsund Wissensorganisationsprogramm Citavi codiert. Zur Beschreibung der Studien wurden Forschungsansatz (gemäss Angabe der Autoren), Untersuchungsinstrumente, Anzahl und Form der jeweils untersuchten Schulen, Zeitpunkt der Studie mit Blick auf die Dauer der Implementation digital gestützten personalisierten Lernens, Untersuchungszeitraum (Quer- oder Längsschnitt) sowie befragte Akteursgruppen codiert.

Zur Beantwortung der ersten Forschungsfrage wurden übergeordnete Ergebnisse zur Implementation digital gestützten personalisierten Lernens in einem Code «Modelle/Implementationen» kategorisiert. Mit Blick auf den Schulentwicklungsprozess (Forschungsfrage 2 und 3) wurde deduktiv ein Kategoriensystem mit den drei Hauptkategorien «Unterrichtsentwicklung», «Personalentwicklung» und "Organisationsentwicklung» angewandt, für die jeweils als Unterkategorien "Barrieren/Herausforderungen/Probleme» und «Gelingensbedingungen/Lösungen» erfasst wurden. Innerhalb dieser Kategorien wurden induktiv weitere Codes gebildet, mit denen die in den Studien berichteten Ergebnisse zusammengefasst wurden. Hierauf wird im Ergebnisteil genauer eingegangen.

\subsection{Beschreibung der eingeschlossenen Studien}

Mehrheitlich handelt es sich bei den eingeschlossenen Studien um Untersuchungen mit komplexen Mixed-Method-Designs, bei denen qualitative Forschungsansätze im Vordergrund stehen (vgl. Tab. 1). Bemerkenswert ist die Vielfalt der eingesetzten Forschungsinstrumente. Dabei stellen mit Ausnahme der Survey-Studien von Lee et al. (2018) und Admiraal et al. (2018) in allen Untersuchungen Interviews eine zentrale Datenquelle dar. In etwa der Hälfte der Studien wurden ausserdem Beobachtungsdaten erhoben, wobei es sich vorrangig um Unterrichtsbeobachtungen handelt. Qualitative Daten wurden weiterhin in Fokusgruppen, durch Dokumentenanalysen, Bilder und Skizzen sowie Logbücher gewonnen. 


\begin{tabular}{|c|c|c|c|c|c|c|}
\hline Studie & Forschungsansatz & Instrumente & $\begin{array}{l}\text { Schulstich- } \\
\text { probe }\end{array}$ & $\begin{array}{l}\text { Dauer der } \\
\text { Implementa- } \\
\text { tion }\end{array}$ & $\begin{array}{l}\text { Untersu- } \\
\text { chungszeit- } \\
\text { raum }\end{array}$ & Befragte \\
\hline $\begin{array}{l}\text { Admiraal et al. } \\
2018\end{array}$ & $\begin{array}{l}\text { Quantitative Studie mit } \\
\text { Kontrollgruppen-Design, } \\
\text { Kovarianzanalyse }\end{array}$ & $\begin{array}{l}\text { Fragebogen, Leistungs- } \\
\text { test, ergänzend Inter- } \\
\text { view, Logbuch }\end{array}$ & $\begin{array}{l}27 \text { Schulen } \\
\text { (Sek I und II) }\end{array}$ & k. A. & Querschnitt & $\begin{array}{l}\text { Lehrkräfte, } \\
\text { SuS }\end{array}$ \\
\hline $\begin{array}{l}\text { Amro und } \\
\text { Borup } 2019\end{array}$ & $\begin{array}{l}\text { Qualitative Inhaltsana- } \\
\text { lyse }\end{array}$ & Interview & k. A. & mind. 1 Jahr & Querschnitt & Lehrkräfte \\
\hline $\begin{array}{l}\text { Basham et al. } \\
2016\end{array}$ & $\begin{array}{l}\text { Mixed Methods (Quali- } \\
\text { tative, deskriptive Be- } \\
\text { obachtung, quantitative } \\
\text { Wachstumsanalysen) }\end{array}$ & $\begin{array}{l}\text { Beobachtung (Unterricht, } \\
\text { Teamsitzungen), Inter- } \\
\text { view, Leistungs- und Sozi- } \\
\text { aldaten der Schülerinnen } \\
\text { und Schüler }\end{array}$ & $\begin{array}{l}12 \text { Schulen } \\
\text { (Primarstufe, } \\
\text { Sek I, Sek II) }\end{array}$ & k. A. & $\begin{array}{l}\text { Längsschnitt } \\
18 \text { Monate }\end{array}$ & $\begin{array}{l}\text { Lehrkräfte, } \\
\text { SuS }\end{array}$ \\
\hline Bingham 2017 & $\begin{array}{l}\text { Qualitative Einzelfall- } \\
\text { studie }\end{array}$ & $\begin{array}{l}\text { Beobachtung (Unterricht, } \\
\text { Teamsitzungen, SchiLf) } \\
\text { Interview, Fokusgruppe }\end{array}$ & $\begin{array}{l}\text { Einzelschule } \\
\text { (Sek I+II,) }\end{array}$ & 1. -3. Jahr & $\begin{array}{l}\text { Längsschnitt } \\
3 \text { Jahre }\end{array}$ & $\begin{array}{l}\text { Lehrkräfte, } \\
\text { Schulleitun- } \\
\text { gen, SuS }\end{array}$ \\
\hline $\begin{array}{l}\text { Bingham et al. } \\
2018\end{array}$ & Vergleichende Fallstudie & $\begin{array}{l}\text { Beobachtung (Unterricht, } \\
\text { Teamsitzungen, SchiLf) } \\
\text { Interview, Fokusgruppe, } \\
\text { Fragebogen }\end{array}$ & \begin{tabular}{|l}
28 Schulen \\
(davon 9 \\
tiefergehend; \\
Primarstufe, \\
Sek I, Sek II) \\
\end{tabular} & 1. und 2. Jahr & $\begin{array}{l}\text { Längsschnitt } \\
18 \text { Monate }\end{array}$ & $\begin{array}{l}\text { Lehrkräfte, } \\
\text { Schulleitun- } \\
\text { gen, SuS }\end{array}$ \\
\hline Coiro et al. 2018 & $\begin{array}{l}\text { Deskriptive strukturierte } \\
\text { Beobachtung }\end{array}$ & $\begin{array}{l}\text { Beobachtung (Unter- } \\
\text { richt), Interview, Frage- } \\
\text { bogen }\end{array}$ & $\begin{array}{l}8 \text { Schulen } \\
\text { (Sek I) }\end{array}$ & k. A. & Querschnitt & $\begin{array}{l}\text { Lehrkräfte, } \\
\text { SuS }\end{array}$ \\
\hline Galagher 2914 & $\begin{array}{l}\text { Mixed Methods (Quan- } \\
\text { titative und qualitative } \\
\text { Befragung) }\end{array}$ & Fragebogen, Interview & $\begin{array}{l}20 \text { Schulen } \\
\text { (Primarstufe, } \\
\text { Sek I, Sek II) }\end{array}$ & k. A. & Querschnitt & $\begin{array}{l}\text { Schulleitun- } \\
\text { gen, Lehkräf- } \\
\text { te, Admin. }\end{array}$ \\
\hline $\begin{array}{l}\text { Galle, Stebler } \\
\text { und Reusser } \\
2019\end{array}$ & $\begin{array}{l}\text { Strukturierte Inhalts- } \\
\text { analyse }\end{array}$ & Interview & $\begin{array}{l}2 \text { Schulen } \\
\text { (Primarstufe) }\end{array}$ & k. A. & $\begin{array}{l}\text { Längsschnitt } \\
3 \text { Jahre }\end{array}$ & $\begin{array}{l}\text { Lehrkräfte, } \\
\text { Schulleitun- } \\
\text { gen }\end{array}$ \\
\hline $\begin{array}{l}\text { Gross und } \\
\text { DeArmond } 2018\end{array}$ & $\begin{array}{l}\text { Mixed Methods, tw. Kont- } \\
\text { rollgruppen-Design }\end{array}$ & $\begin{array}{l}\text { Beobachtung (Unterricht) } \\
\text { Interview, Fokusgruppe, } \\
\text { Fragebogen }\end{array}$ & $\begin{array}{l}39 \text { Schulen } \\
\text { (Primarstufe, } \\
\text { Sek I, Sek II) }\end{array}$ & 1. und 2. Jahr & Querschnitt & $\begin{array}{l}\text { Lehrkräfte, } \\
\text { Schulleitun- } \\
\text { gen, Adminis- } \\
\text { tration }\end{array}$ \\
\hline Lee et al. 2018 & Survey & Fragebogen & $\begin{array}{l}41 \text { Schulen } \\
\text { (Primarstufe, } \\
\text { Sek I, Sek II) }\end{array}$ & mind. 2. Jahr & Querschnitt & Lehrkräfte \\
\hline Kallio 2018 & Phänomenologie & $\begin{array}{l}\text { Beobachtung (Raum), } \\
\text { Bilder, Interview, Fokus- } \\
\text { gruppe }\end{array}$ & $\begin{array}{l}\text { 4 Schulen } \\
\text { (Primarstufe, } \\
\text { Sek I, Sek II) }\end{array}$ & k. A. & Querschnitt & $\begin{array}{l}\text { Lehrkräfte, } \\
\text { SuS }\end{array}$ \\
\hline Myers 2018 & $\begin{array}{l}\text { Einzelfallstudie } \\
\text { Phänomenologie }\end{array}$ & Interview, Fokusgruppe & $\begin{array}{l}\text { Einzelschule } \\
\text { (Sek I) }\end{array}$ & 3. Jahr & Querschnitt & $\begin{array}{l}\text { Lehrkräfte, } \\
\text { Schulleitung, } \\
\text { Administra- } \\
\text { tion }\end{array}$ \\
\hline $\begin{array}{l}\text { Netcoh und } \\
\text { Bishop } 2017\end{array}$ & Einzelfallstudie & $\begin{array}{l}\text { Beobachtung (Unterricht) } \\
\text { Interview, Fokusgruppe, } \\
\text { Dokumentenanalyse }\end{array}$ & $\begin{array}{l}\text { Einzelschule } \\
\text { (Sek I) }\end{array}$ & 1. Jahr & Querschnitt & Lehrkräfte \\
\hline Pane et al. 2017 & $\begin{array}{l}\text { Mixed Methods, tw. Kont- } \\
\text { rollgruppen-Design }\end{array}$ & $\begin{array}{l}\text { Beobachtung (Unterricht) } \\
\text { Interview, Fokusgruppe, } \\
\text { Fragebogen, standardis. } \\
\text { Schulleitungstest }\end{array}$ & $\begin{array}{l}40 \text { Schulen } \\
\text { (Primarstufe, } \\
\text { Sek I, Sek II;) }\end{array}$ & 1.-3. Jahr & $\begin{array}{l}\text { Längsschnitt } \\
3 \text { Jahre }\end{array}$ & $\begin{array}{l}\text { Lehrkräfte, } \\
\text { Schulleitun- } \\
\text { gen }\end{array}$ \\
\hline Prain et al. 2013 & Mixed Methods & $\begin{array}{l}\text { Interview, Fragebogen, } \\
\text { Leistungstest }\end{array}$ & $\begin{array}{l}\text { 4 Schulen } \\
\text { (Sek I) }\end{array}$ & k. A. & Querschnitt & $\begin{array}{l}\text { Lehrkräfte, } \\
\text { Schulleitun- } \\
\text { gen, SuS }\end{array}$ \\
\hline $\begin{array}{l}\text { Staub, Stebler } \\
\text { und Reusser } \\
2018\end{array}$ & $\begin{array}{l}\text { Qualitative Inhaltsana- } \\
\text { lyse }\end{array}$ & $\begin{array}{l}\text { Interview, Dokumenten- } \\
\text { analyse }\end{array}$ & 12 Schulen & 1.-3. Jahr & $\begin{array}{l}\text { Längsschnitt } \\
3 \text { Jahre }\end{array}$ & $\begin{array}{l}\text { Lehrkräfte, } \\
\text { Schulleitun- } \\
\text { gen }\end{array}$ \\
\hline Taylor 2016 & $\begin{array}{l}\text { Qualitative Inhaltsana- } \\
\text { lyse }\end{array}$ & Interview & $\begin{array}{l}\text { Einzelschule } \\
\text { (Sek. I) }\end{array}$ & 1. Jahr & $\begin{array}{l}\text { Längsschnitt } \\
6 \text { Monate }\end{array}$ & Lehrkräfte \\
\hline $\begin{array}{l}\text { Yildirim et al. } \\
2014\end{array}$ & $\begin{array}{l}\text { Qualitative Inhaltsana- } \\
\text { lyse }\end{array}$ & $\begin{array}{l}\text { Interview, Beobachtung, } \\
\text { Dokumentenanalyse }\end{array}$ & $\begin{array}{l}9 \text { Schulen } \\
\text { (Vorschule, } \\
\text { Primarstufe, } \\
\text { Sek I, Sek II) }\end{array}$ & $\begin{array}{l}\text { Mehrere } \\
\text { Jahre }\end{array}$ & Querschnitt & $\begin{array}{l}\text { Lehrkräfte, } \\
\text { Schulleitun- } \\
\text { gen, Medien- } \\
\text { koordinato- } \\
\text { ren }\end{array}$ \\
\hline
\end{tabular}

Tab. 1.: Eingeschlossene Studien. 
Fragebögen wurden, teilweise als primäres Instrument, überwiegend jedoch eher ergänzend, in etwa einem Drittel der Studien eingesetzt. In drei Studien wurden auf dieser Grundlage quasi-experimentelle quantitative Vergleiche mit «traditionellen» Schulen angestellt (Admiraal et al. 2018; Gross und DeArmond 2018; Pane et al. 2017). Für quantitative Analysen auf der Ebene der Schülerinnen und Schüler wurden darüber hinaus Daten aus Schulleistungstests, Einstellungs- und Motivationsfragebögen sowie schulstatistische deskriptive Daten herangezogen (Admiraal et al. 2018; Basham et al. 2016; Pane et al. 2017).

In den meisten Fällen wurden die qualitativen Daten mit inhaltsanalytischen Verfahren induktiv ausgewertet. Einige Studien (Admiraal et al. 2018; Bingham et al. 2018; Bingham 2017; Coiro et al. 2018; Lee et al. 2018; Staub, Stebler und Reusser 2018) gehen deduktiv-induktiv vor, wobei unterschiedliche Modelle (z. B. kulturhistorische Aktivitätstheorie, unterschiedliche Modelle personalisierten Lernens) zugrunde gelegt wurden.

Hinsichtlich der Schulstichproben variieren die eingeschlossenen Studien von Untersuchungen an Einzelschulen bis zu Untersuchungen mit mehr als 40 Schulen. Bei den Untersuchungen mit grösseren Schulstichproben handelt es sich häufig um Evaluationen von Pilotversuchen bzw. von staatlich und privat geförderten Initiativen zur Stärkung des personalisierten Lernens (Basham et al. 2016; Bingham et al. 2018; Coiro et al. 2018; Gross und DeArmond 2018; Pane et al. 2017; Reusser, Pauli und Stebler 2018). Einige Studien stellen vertiefende Analysen an Teilstichproben solcher breiter angelegten Evaluationen dar (Bingham 2017; Galle, Stebler und Reusser 2019; Kallio 2018; Staub, Stebler und Reusser 2018). Überwiegend untersuchen die Studien Schulentwicklungsprozesse der ersten zwei bis drei Jahre nach der Einführung des personalisierten Lernens. Dabei erfolgte die Datenerhebung etwa an der Hälfte der Studien an einem singulären Messzeitpunkt und bildet den Schulentwicklungsprozess querschnittlich ab. Die vorliegenden längsschnittliche Erhebungen erstrecken sich über einen Zeitraum von 6 Monaten bis zu drei Jahren.

Mit Ausnahme von Lee et al. (2018) sowie Netcoh und Bishop (2017) beziehen die Untersuchungen mehrere Akteursgruppen ein, allen voran Lehrpersonen, ausserdem Mitglieder von Schulleitungen und Schuladministration sowie Schülerinnen und Schüler. Die Stichproben variierten dabei erheblich von Einzelfallanalysen einzelner Lehrerinnen und Lehrer (Myers 2018) bis zur Fragebogen-Befragung bei mehreren 1.000 Lehrkräften (Gross und DeArmond 2018).

Von wenigen Untersuchungen abgesehen (Admiraal et al. 2018; Galle, Stebler und Reusser 2019; Prain et al. 2013; Staub, Stebler und Reusser 2018), wurden die Studien an Schulen in den USA durchgeführt. Im deutschen Sprachraum konnte nur die PerLEN-Studie von Reusser, Pauli und Stebler (2018) lokalisiert werden (s. a. Reusser, Pauli und Stebler 2018; Galle, Stebler und Reusser 2019; Staub, Stebler und Reusser 2018). 
Zusammenfassend lässt sich feststellen, dass die Majorität der Studien durch ihre komplexen Designs, die meist unterschiedliche Messinstrumente und mehrere Akteursgruppen einbeziehen, um eine umfassende, ganzheitliche und multiperspektivische Analyse von Schulentwicklungsprozessen bemüht sind. Da nur ein Teil der Studien ein Längsschnitt-Design aufweist, werden Prozesse und Entwicklungen häufig eher retrospektiv von den Beteiligten rekonstruiert. Bei der Interpretation der nachfolgend dargestellten Ergebnisse ist auch zu berücksichtigen, dass die vorliegenden Studien vorwiegend die Einführung personalisierten Lernens sowie die Etablierung des Konzepts in den Anfangsjahren beschreiben und nur bedingt Aussagen über eine längerfristige Implementation personalisierten Lernens erlauben. Schliesslich sollte im Blick behalten werden, dass die Einführung an US-amerikanischen Schulen aufgrund sich deutlich unterscheidender bildungspolitischer Rahmenbedingungen und auch einer meistens erheblich längeren Tradition des Einsatzes digitaler Medien an der Schule nicht ohne Weiteres auf die Verhältnisse an Schulen im deutschen Sprachraum übertragen werden kann (s. u.).

\section{Ergebnisse}

\subsection{Implementierung personalisierten Lernens}

Eingangs wurde dargestellt, dass personalisiertes Lernen ein breites Spektrum unterschiedlicher Formen annehmen kann. Die vorliegenden Studien dokumentieren die grosse Heterogenität der Ansätze, die von der Einführung von Freiarbeitsbändern für projektbasiertes Arbeiten (Netcoh und Bishop 2017) bis zur durchgängigen Implementierung eines adaptiven Lern-Managementsystems mit weitreichenden Diagnose- und Unterstützungswerkzeugen für Schülerinnen und Schüler sowie Lehrkräfte reicht (Amro und Borup 2019).

Unterschiedliche Implementationsmodelle

Nur wenige Studien haben den Versuch unternommen, die Vielfalt der Implementationen zu systematisieren. So unterteilen Coiro et al. (2018) die von ihnen untersuchten Schulen grob in «Blended-Learning-Schulen», die eher offene, projektbasierte Umsetzungen personalisierten Lernens umsetzen und «Summit-Schulen», die mit der gleichnamigen adaptiven Lernplattform arbeiten und den Schülerinnen und Schülern eher stark vorstrukturierte, individualisierte Lernwege bieten.

Gallagher (2014) identifiziert auf der Grundlage einer Befragung von 20 Administratorinnen und Administratoren zwei Modelle: Schulen, an denen die Technologie innerhalb des Unterrichts zur Differenzierung eingesetzt wird und Schulen, an denen die Lernenden überwiegend ausserhalb des Unterrichts zuhause oder im Selbststudium mit einer Lernplattform arbeiten. 
Admiraal et al. (2018) legen ein dreidimensionales Raster an, mit dem sie die Personalisierungsansätze - auf der Ebene einzelner beobachteter Schulklassen - in lehrkraftseitige divergente vs. konvergent-homogene vs. konvergent-heterogene Differenzierung, schülerseitiges Ausmass der Steuerung des Lernprozesses (hohe vs. geringe Lernendenkontrolle) und systemseitige Adaptivität des eingesetzten digitalen Lernprogramms (hoch vs. niedrig) unterscheiden. Leider machen sie jedoch keine Angabe dazu, wie häufig die verschiedenen Merkmale und Merkmalskombinationen in ihrer Stichprobe von 27 Schulen vorkommen.

Das umfassendste System zur Kategorisierung stammt von Pane et al. (2017), die auf der Grundlage der Analysen an 40 Schulen, die im Rahmen der Next Generation Learning Challenge (NGLC) der Bill und Melinda Gates Stiftung gefördert wurden, vier (interdependente) idealtypische Implementierungsstrategien unterscheiden:

1. Lernendenprofile: Individuelle Stärken, Bedürfnisse, Motivation, Lernfortschritte und Ziele der Schülerinnen und Schüler werden möglichst unter Einbezug aller verfügbaren Datenquellen transparent dokumentiert und möglichst allen schulischen Akteuren (Lehrkräfte, Schülerinnen und Schüler, Eltern) zur Verfügung gestellt. Die Lernendenprofile werden regelmässig aktualisiert und mit den Schülerinnen und Schülern reflektiert, um individuelle Lernpläne festzulegen. Die Schule hält eine Vielfalt von Lernmaterialien bereit, um den individuellen Lernbedürfnissen ihrer Schülerinnen und Schüler gerecht zu werden. Während des Schultags gibt es regelmässige Zeitfenster für Einzelberatung und individuelle Lernunterstützung durch die Lehrkräfte.

2. Kompetenzbasierte Lernprogression: Die Schülerinnen und Schüler bearbeiten das Lernmaterial entsprechend ihrer Fähigkeiten in ihrem eigenen Tempo und in individualisierter Abfolge. Lernfortschritte werden regelmässig kompetenzbasiert und bedarfsgerecht überprüft, wobei unterschiedliche Bewertungsformate zum Einsatz kommen und die erreichten Kompetenzen durch Punkte oder credits dokumentiert werden.

3. Flexible Lernumgebungen: Weitreichende Flexibilität beim Einsatz personeller, zeitlicher und räumlicher Ressourcen. Beispielsweise kann der Schultag flexibel gestaltet oder die Schülerinnen und Schüler flexibel, auch jahrgangsübergreifend, gruppiert werden. Ausgangspunkt sind die Bedürfnisse der Lernenden. Die Flexibilisierung erfolgt datengestützt und wird durch den Einsatz digitaler Endgeräte unterstützt.

4. Persönliche Lernpfade: Diese werden auf der Grundlage der individuellen Lernendenprofile entwickelt. Innerhalb von durch die Lehrkräfte festgelegten Parametern erhalten die Schülerinnen und Schüler Freiräume bei der Bearbeitung von Lernaufgaben. 
(Geringe) Unterschiede zwischen Projekt- und Vergleichsschulen

Die vergleichende Lehrkräftebefragung von Pane et al. (2017) zeigt, dass an den NGLCSchulen im Vergleich zu Schulen, die nicht an dem Programm teilnehmen, stärker auf Schülerinnen und Schülerdaten und -bewertungen bei der Individualisierung des Lernens zurückgegriffen wird und auch häufiger digitale Lernsysteme genutzt werden. Auch mit Blick auf die Flexibilisierung der Lernumgebung schneiden die NGLCSchulen besser ab. Andererseits konnte nicht festgestellt werden, dass die Schülerinnen und Schüler an den Projektschulen mehr Autonomie über ihren Lernprozess erhalten (vgl. auch Coiro et al. 2018), dass die Lernangebote grundsätzlich stärker auf ihre Bedürfnisse zugeschnitten sind oder dass sie häufiger individuelle Lernberatung erhalten. Pane et al. (2017) räumen deshalb ein, dass sich die Projektschulen insgesamt nicht so deutlich von «traditionellen» Schulen unterscheiden, wie der Ansatz vermuten lassen würde. Noch kritischer urteilen Gross und DeArmond (2018) über die von ihnen untersuchten Schulen (ebenfalls Teilnehmende der Next Generation Learning Challenge). Zwar finden auch sie im Vergleich mit einer Referenzstichprobe, dass Lehrkräfte an Projektschulen häufiger angeben, verschiedene Grundsätze personalisierten Lernens umzusetzen als Lehrpersonen von nicht an dem Programm teilnehmenden Schulen. Allerdings kommen sie in der Zusammenführung der Ergebnisse aus Unterrichtsbeobachtungen, Interviews und Fragebögen zu dem Ergebnis, dass die Personalisierung des Lernens mehrheitlich oberflächlich, uneinheitlich und inkonsequent umgesetzt wird (vgl. auch Coiro et al. 2018; Gallagher 2014; Netcoh und Bishop 2017).

Erhebliche Varianz der Implementationen

In der Zusammenschau der Studien scheint es insgesamt so zu sein, dass die Implementation personalisierten Lernens selbst an Projektschulen bislang häufig auf der Ebene einzelner Lehrkräfte und/oder Schulklassen erfolgt. Dabei scheint ein übergreifender einheitlicher Ansatz personalisierten Lernens eher die Ausnahme zu sein. Vielmehr bestehen in den meisten dokumentierten Implementationen - selbst innerhalb einer Schule, erst recht aber zwischen verschiedenen Schulen, die an demselben Programm teilnehmen - eine Vielzahl didaktischer Ansätze, Methoden, Tools und Lernorganisationen nebeneinander. Dieses mag auch der Tatsache geschuldet sein, dass in der Anfangsphase bewusst Freiräume geschaffen werden, um unterschiedliche Möglichkeiten personalisierten Lernens und ihre Potenziale zu erproben. Ein fehlendes Gesamtkonzept führt jedoch, gerade innerhalb der Einzelschule, zu verschiedenen Herausforderungen, auf die im Folgenden genauer eingegangen wird. 


\subsection{Personalisiertes digital gestütztes Lernen und Unterrichtsentwicklung}

\section{Mehrwert: Lernprozessbezogene Daten der Schülerinnen und Schüler}

Als bedeutender Mehrwert digital gestützten personalisierten Lernens auf der Unterrichtsebene werden in vielen Studien die durch personalisierte Lernprogramme und -plattformen bereitgestellten Schülerinnen- und Schülerdaten herausgestellt (Amro und Borup 2019; Bingham 2017; Coiro et al. 2018; Pane et al. 2017; Yildirim et al. 2014). Im Vordergrund stehen dabei Daten zum Lernfortschritt (Amro und Borup 2019; Bingham 2017; Lee et al. 2018). Aber auch weitere Daten, z. B. zu Lernzeiten, Arbeitsmustern, Interessen und Vorlieben werden von Lehrkräften als hilfreich empfunden (Amro und Borup 2019; Lee et al. 2018; Yildirim et al. 2014). Die Daten werden genutzt, um Schülerinnen und Schülern zeitnahe und differenzierte Rückmeldungen und Lernberatung anbieten zu können (Bingham 2017; Pane et al. 2017; Lee et al. 2018). Auch die Schülerinnen und Schüler selbst nutzen diese Daten, um ihren Lernfortschritt zu reflektieren (Pane et al. 2017). Die Daten erhöhen dabei die Transparenz der Bewertung und Einschätzung von Lernfortschritten, sowohl in der Kommunikation von Lehrkräften und Lernenden, als auch von Lehrkräften untereinander sowie zwischen Lehrkräften und Eltern (Amro und Borup 2019; Basham et al. 2016). Die Vergleichsuntersuchung von Pane et al. (2017) zeigt allerdings, dass die Bereitstellung digitaler Daten, wie bereits erwähnt, nicht dazu führt, dass Lehrkräfte ihre Lernangebote wesentlich stärker auf die Bedürfnisse der Schülerinnen und Schüler zuschneiden als Lehrkräfte, denen solche Daten nicht zur Verfügung stehen.

Mehrwert: Differenzierung und Flexibilisierung von Lernwegen

Dennoch ist die Differenzierung und Flexibilisierung von Lernwegen ein weiterer häufig genannter Mehrwert des digital gestützten personalisierten Lernens (Basham et al. 2016; Coiro et al. 2018; Myers 2018; Netcoh und Bishop 2017; Lee et al. 2018). Allerdings schränken einige Studien (Coiro et al. 2018; Pane et al. 2017) ein, dass Schülerinnen und Schüler nur relativ begrenztes Mitspracherecht bei der Wahl von Lerninhalten und Formaten bzw. nur oberflächliche Wahlmöglichkeiten erhalten (z. B. wann eine Hausaufgabe abgegeben oder welche von mehreren Aufgaben eingereicht wird).

Mehrwert: Verbesserung der Beziehung von Lehrkräften und Lernenden

Eine dritte positive Konsequenz auf Unterrichtsebene, die mehrfach berichtet wird, ist eine verbesserte Beziehung zwischen Lehrkräften und ihren Schülerinnen und Schülern (Coiro et al. 2018; Myers 2018; Netcoh und Bishop 2017). Dies begründet sich aus neuen Unterrichtsstrukturen, in denen Lehrkräfte intensiver mit einzelnen Schülerinnen und Schülern arbeiten (Myers 2018; Netcoh und Bishop 2017). Aber 
auch die zusätzlich verfügbaren Daten (s. o.) ermöglichen es den Lehrkräften, ihre Schülerinnen und Schüler genauer kennenzulernen und besser einschätzen zu können (Coiro et al. 2018).

Eigenverantwortliches Lernen als Lernaufgabe für Lehrkräfte sowie Schülerinnen und Schüler

Die Mehrzahl der berichteten Schwierigkeiten und Herausforderungen in der Unterrichtsentwicklung resultieren aus einer Veränderung der Unterrichtspraxis, die den Schülerinnen und Schülern grössere Freiräume zugesteht, ihnen aber auch mehr Verantwortung für ihren Lernprozess abverlangt. Typischerweise scheint es dabei zunächst zu einer Überforderung der Lernenden zu kommen, indem ihnen ein grosses (und wenig differenziertes) Mass an Eigenverantwortung übertragen wird und sie nur ungenügende Unterstützung in Form von Zielvereinbarungen, Rückmeldungen, lernstrategischen Hilfen usw. erhalten. Auch wenn in den verwendeten Lernprogrammen und -plattformen individuelle Ziele, Rückmeldungen und Unterstützungen festgelegt werden (können), erweist sich die personalisierte Lernunterstützung durch die Lehrkräfte als unverzichtbar. Besonders plastisch beschreibt Bingham (2017) diese Erfahrung von Lehrkräften an einer Brennpunktschule im ersten Jahr der Implementierung einer Plattform für personalisiertes Lernen. Aber auch Netcoh und Bishop (2017), Pane et al. (2017), Prain et al. (2013), Taylor (2016) sowie Basham et al. (2016) berichten, dass Lehrkräfte (zunächst) Schwierigkeiten hatten, personalisiertes Lernen so zu gestalten, dass gerade auch weniger motivierte oder leistungsschwächere Schülerinnen und Schüler erfolgreich selbstgesteuert lernten. In diesem Zusammenhang kritisieren Gross und DeArmond (2018) auf der Basis von Unterrichtsbeobachtungen, dass die Reflektion verschiedener Lernpfade und Lernentscheidungen im Unterricht häufig zu kurz kommt. Der Entwicklungsprozess der Lehrkräfte, der in unterschiedlichen Studien sichtbar wird, kreist dabei zum einen um das Problem, das jeweils für die Schülerinnen und Schüler angemessene Mass von Freiraum und Führung bei der Strukturierung ihrer Lernprozesse zu finden. Dies zeigt sich z. B. daran, dass Lehrkräfte in mehreren Studien berichten, dass es ihnen schwer fällt abzuwägen, wieviel strukturelle und zeitliche Vorgaben Schülerinnen und Schüler für die Bewältigung von Lernaufgaben benötigen (Netcoh und Bishop 2017; Pane et al. 2017). Auch die Schülerinnen und Schüler in der Befragung von Prain et al. (2013) geben an, dass sie ihre Lehrerinnen und Lehrer als unsicher hinsichtlich des Grads an Offenheit und Führung im Unterricht erleben. Zum anderen wird bemängelt, dass individualisiertes Lernen mit digitalen Medien zu häufig isoliert erfolgt und nicht ausreichend mit Phasen, in denen die Schülerinnen und Schüler individuell oder in Kleingruppen durch die Lehrkräfte beraten werden, verbunden wird (Amro und Borup 2019). Als Grund hierfür nennen die Lehrkräfte in der Studie von Amro und Borup (2019) fehlende Vorbereitungszeit, um sich mit den je individuellen Lernständen ihrer Schülerinnen und 
Schüler auseinanderzusetzen, unübersichtliche und unklare Rückmeldungen der genutzten Programme sowie fehlende Fortbildung (s. u.). Schliesslich kritisieren Gross und DeArmond (2018) die Personalisierung auch deshalb als unzureichend, weil vor allem leistungsstärkere Schülerinnen und Schüler herausfordernde und interessante Projektaufgaben erhalten, während ihre leistungsschwächeren Klassenkameradinnen und -kameraden an wenig motivierenden Übungsaufgaben arbeiten.

Unterrichtsorganisation als Herausforderung

Herausforderungen stellen sich weiterhin in unterrichtsorganisatorischer Hinsicht. So erweist sich die zunehmende Asynchronität der Lernprozesse der Schülerinnen und Schüler als Problem, wenn trotz unterschiedlicher Lernzeiten dennoch gemeinsame zeitliche Strukturen (z. B. Abgabetermine) eingehalten werden sollen (Netcoh und Bishop 2017; Pane et al. 2017) oder an gemeinsamen Lernaufgaben gearbeitet werden soll (Pane et al. 2017). Auch das Management der unterschiedlichen Lernpfade, denen die Schülerinnen und Schüler folgen, wird in einigen Studien als aufwändig für die Lehrkräfte beschrieben (Amro und Borup 2019; Netcoh und Bishop 2017; Taylor 2016). Die Vielzahl an Lernpfaden und -optionen können dabei auch für die Schülerinnen und Schüler verwirrend sein (Gross und DeArmond 2018; Taylor 2016). Nicht alle Studien bestätigen jedoch die genannten unterrichtsorganisatorischen Probleme. So finden Coiro et al. (2018), dass Schülerinnen und Schüler insbesondere an den Summit Schulen häufig sowohl Gelegenheiten für individuelles als auch kollaboratives Lernen erhalten. Auch Basham et al. (2016) berichten eine grosse Variabilität und Flexibilität an Lernangeboten ohne die genannten Probleme.

Strukturen, Zielsetzungen, Rechenschaftslegung und Verantwortung als Erfolgsbedingungen

Als bedeutende Erfolgsfaktoren insbesondere hinsichtlich der Öffnung des Unterrichts kristallisieren sich in mehreren Studien klare Strukturen und Zielsetzungen durch die Lehrpersonen sowie Verantwortung und Rechenschaftslegung für die individuellen Lernprozesse auf Seiten der Schülerinnen und Schüler heraus (Amro und Borup 2019; Basham et al. 2016; Bingham 2017; Coiro et al. 2018; Pane et al. 2017). Strukturierung und Zielsetzungen beziehen sich dabei z. B. darauf, dass (gemeinsam mit den Schülerinnen und Schülern erarbeitete) Vorgaben gemacht werden, wann und wie lange mit personalisierten Lernsystemen gelernt werden soll, welche Einheiten bearbeitet oder wie viele Punkte erreicht werden sollen (Amro und Borup 2019; Bingham 2017). Verantwortung und Rechenschaftslegung sind mit diesen Zielsetzungen unmittelbar verknüpft, indem systemgenerierte Daten genutzt werden, um mit den Schülerinnen und Schülern ihre Lernfortschritte zu reflektieren und ihnen zurückzuspiegeln, wie weit sie ihre Lernziele erreicht haben (Bingham 2017; Prain et al. 2013). 


\subsection{Personalisiertes digital gestütztes Lernen und Personalentwicklung}

Zwar finden Coiro et al. (2018) in ihrer Befragung, dass ein Grossteil der Lehrkräfte der Meinung ist, verschiedene Dimensionen personalisierten Lernens erfolgreich umsetzen zu können und somit seine Kompetenzen ausgesprochen positiv einschätzt. Dennoch wird mit Blick auf die Kompetenzen der Lehrpersonen in den vorliegenden Untersuchungen vielfältiger Personalentwicklungsbedarf konstatiert. Zunächst stellen zahlreiche Studien fest, dass personalisiertes Lernen für sie im Widerspruch zu ihrer bisherigen Unterrichtspraxis steht (Bingham et al. 2018; Coiro et al. 2018; Gross und DeArmond 2018; Netcoh und Bishop 2017; Taylor 2016). Dies betrifft vor allem die im vorigen Abschnitt bereits beschriebene Notwendigkeit zur Öffnung des Unterrichts, die eine Veränderung der Strukturen und Abläufe im Unterricht, der zu fördernden Kompetenzen bei den Schülerinnen und Schülern und nicht zuletzt der Lehrpersonenrolle mit sich bringt. Lehrkräfte haben dabei Schwierigkeiten, das abstrakte Ziel einer personalisierten Lernförderung in konkrete Unterrichtshandlungen und -abläufe zu übersetzen (Gross und DeArmond 2018).

\section{Diagnostische Kompetenzdefizite}

Kompetenzdefizite der Lehrkräfte, die vor diesem Hintergrund festgestellt werden, betreffen zum einen ihre diagnostische Kompetenz, also die Fähigkeit festzustellen und zu entscheiden, welche Schülerinnen und Schüler welche Art von Lern- und Unterstützungsangeboten benötigen (Netcoh und Bishop 2017). In diesem Zusammenhang merken Pane et al. (2017) an, dass Lehrpersonen Schwierigkeiten haben, die Fülle an Daten, die digitale personalisierte Lernprogramme und -plattformen für die Diagnostik zur Verfügung stellen, zu interpretieren und daraus Schlussfolgerungen für ihren Unterricht abzuleiten (vgl. auch Amro und Borup 2019).

\section{Methodisch-didaktische Kompetenzdefizite}

Weiterhin werden mehrfach methodisch-didaktische Kompetenzdefizite angesprochen, etwa in der Planung und Durchführung offener Unterrichtsformen wie Projektunterricht (Bingham et al. 2018). Ausserdem fehlten den Lehrpersonen methodische Strategien zur Förderung von Selbständigkeit und Autonomie der Schülerinnen und Schüler (Bingham 2017; Gross und DeArmond 2018) sowie Wissen darüber, wie digitale Medien sinnvoll in den Unterricht eingebunden werden können (Bingham et al. 2018). Schliesslich verfügen viele Lehrkräfte nicht über das notwendige Wissen hinsichtlich der individualisierten Bewertung von Schülerinnen und Schülern (ebd.).

Negative Einstellungen von Lehrkräften zum personalisierten Lernen Neben Kompetenzdefiziten werden in der Untersuchung von Prain et al. (2013) negative Einstellungen von Lehrkräften zum personalisierten Lernen als Hindernis berichtet. Insbesondere wurde in dieser Studie eine fehlende Bereitschaft der Lehrpersonen 
festgestellt, persönliche Verantwortung für die Lernfortschritte ihrer Schülerinnen und Schüler zu übernehmen. Auch Taylor (2016) stellt fest, dass Überzeugungen von Lehrkräften zum Lehren und Lernen entscheidend dafür sind, ob und in welcher Art und Weise sie bereit sind, personalisiertes Lernen in ihrem Unterricht umzusetzen.

\section{Bedeutung von Fortbildungen}

Fortbildungen sind in verschiedenen Studien erfolgreich, um die genannten Kompetenzdefizite zu kompensieren (Coiro et al. 2018; Myers 2018; Prain et al. 2013). Das Fehlen von Fortbildungen erweist sich dagegen als Hürde bei der Umsetzung (Amro und Borup 2019; Gallagher 2014; Gross und DeArmond 2018). So zeigt sich in der Untersuchung Coiro et al. (2018), dass Lehrkräfte, die an Fortbildungen teilgenommen haben, insgesamt selbstsicherer und aufgeschlossener mit Blick auf digital gestütztes personalisiertes Lernen sind als ihre Kolleginnen und Kollegen, die sich nicht fortgebildet haben. Die Einzelfallstudie von Myers (2018) verdeutlicht in diesem Zusammenhang die Relevanz personalisierter Lernangebote nicht nur auf Seiten der Schülerinnen und Schüler, sondern auch auf Seiten der Lehrpersonen. Auch diese bringen sehr unterschiedliche Voraussetzungen und Vorkenntnisse für die Weiterentwicklung ihres Unterrichts hin zu einer digital gestützten personalisierten Lernumgebung mit und profitieren entsprechend von einem differenzierten Fortbildungsangebot.

\section{Kollegiale Unterstützung}

Abgesehen von Fortbildungen erweist sich die kollegiale Unterstützung im Team als wichtige Personalentwicklungsmassnahme (Coiro et al. 2018; Galle, Stebler und Reusser 2019; Prain et al. 2013). Lehrkräfte legen dabei Wert auf formalisierte Strukturen, die einen regelmässigen und verlässlichen Austausch zulassen (Coiro et al. 2018). Eine effektive Unterstützungsstrategie war in dieser Untersuchung die gegenseitige Unterrichtshospitation (ebd.). Auch Galle, Stebler und Reusser (2019) stellen in ihrer Analyse der Kooperationsstrukturen an zwei Grundschulen, die das Konzept des personalisierten Lernens erfolgreich implementiert haben, fest, dass die qualitativ anspruchsvolle Form der ko-konstruktiven Kooperation zwischen den Lehrkräften (z. B. gemeinsame Unterrichtsplanung, kollegiales Feedback) an diesen Schulen vor anderen, eher oberflächlichen Formen der Kooperation überwiegt.

\section{Freiräume für Erprobungen}

Eine Schwierigkeit für Personalentwicklungsmassnahmen besteht, wie Bingham et al. (2018) und Gross und DeArmond (2018) anmerken, darin, dass aufgrund fehlender Modelle und Erfahrungswerte die o. g. Kompetenzdefizite nicht vollständig durch Schulungen und Fortbildungen adressiert werden können. Die Kompetenzentwicklung erfolgt deshalb häufig durch eigenes wiederholtes Erproben und Ausprobieren - hierfür fehlen den Lehrkräften jedoch an vielen Schulen entsprechende Strukturen 
und Freiräume, die solche wiederholten Erprobungen zulassen (Gross und DeArmond 2018). Lehrkräfte fühlen sich deshalb mit der Umsetzung personalisierten Lernens allein gelassen und überfordert, insbesondere, wenn die o. g. Teamstrukturen fehlen (ebd.).

\section{Fehlende Evaluationskriterien}

Ein weiteres Problem, auf das Gross und DeArmond (2018) hinweisen, besteht darin, dass standardisierte Unterrichtsevaluationen, mit denen die Arbeit von Lehrkräften an Schulen bewertet wird, die besonderen Erfordernisse des personalisierten Lernens nicht abbilden. Damit werden Lehrkräfte, die neue und innovative Unterrichtsmethoden erprobten, durch die etablierten Evaluationssysteme benachteiligt. Zwar fanden an vielen der von Gross und DeArmond (2018) untersuchten Schulen entsprechende Anpassungen der Evaluationsinstrumente statt. War dies jedoch nicht der Fall, äusserten Lehrkräfte Bedenken, dass sie für die Einführung neuer Unterrichtsmethoden am Ende durch negative Evaluationsergebnisse bestraft würden.

\subsection{Personalisiertes digital gestütztes Lernen und Organisationsentwicklung}

Technische Funktionalität von personalisierten Lernplattformen und -programmen Von zentraler Bedeutung für die Umsetzung digital gestützten personalisierten Lernens sind zunächst die technischen Ressourcen, insbesondere die jeweils genutzten digitalen Plattformen und Programme. Lehrkräfte schätzen besonders solche Programme, die ihnen sowie den Schülerinnen und Schülern konkrete, intuitiv verständliche Rückmeldungen zum Lernfortschritt und handlungsnahe Hinweise für weitere Lernaktivitäten geben (Amro und Borup 2019; Basham et al. 2016; Yildirim et al. 2014). Dabei legen sie Wert auf die Möglichkeit, Programme und Plattformen individuell zu konfigurieren und bedarfsgerecht zu adaptieren (Yildirim et al. 2014). Positiv bewertet werden auch Funktionen, die den Unterricht unterstützen, beispielsweise automatisierte Vorschläge für die Gruppierung der Lernenden entsprechend ihres Leistungsstands, integrierte Materialien und Vorschläge für den Unterricht oder eine Anzeige von Schülerinnen und Schülern, die längere Zeit untätig sind (Amro und Borup 2019). Insgesamt fällt auf, dass grundlegende technische Probleme mit Hardund Software in den vorliegenden Untersuchungen kaum berichtet werden. Auf eine überdurchschnittliche Ausstattung deutet die Untersuchung von Pane et al. (2017) hin, in der Lehrkräfte des Next Generation Learning Challenge Programms mit der technische Infrastruktur (Hard- und Software, technischer Support) durchgängig zufriedener waren als Lehrkräfte der Vergleichsgruppe. 
Dennoch zeigen die vorliegenden Untersuchungen zahlreiche Schwachstellen und Entwicklungspotenziale personalisierter digitaler Lernumgebungen und Programme auf. In der breit angelegten Lehrkräftebefragung von Lee et al. (2018) stellt sich heraus, dass selbst Lehrkräfte an Schulen, deren pädagogischer Schwerpunkt auf personalisiertem Lernen liegt, digitale Technologien vor allem zur Planung und Unterstützung des Unterrichts einsetzen. Zur Dokumentation von individuellen Lernfortschritten und zur Bewertung der Schülerinnen und Schüler werden sie hingegen weniger häufig genutzt, da dies aus Sicht der Lehrkräfte durch die vorhandenen Programme nur unzureichend unterstützt wird. Auch verschiedene andere Studien deuten - trotz der oben genannten positiven Bewertungen - darauf hin, dass die bisherigen Plattformen und Programme noch weit davon entfernt sind, Daten bereitzustellen, die eine umfassende, ganzheitliche Diagnostik und Bewertung der Schülerinnen und Schüler ermöglichen. So wird in den Studien von Lee et al. (2018), Pane et al. (2017) sowie Yildirim et al. (2014) kritisiert, dass die bestehenden Plattformen und Programme überwiegend Leistungsdaten erfassen und nicht-leistungsbezogene Merkmale, wie Interessen, sozio-emotionale Fähigkeiten, Verhalten, Anwesenheit usw. zu wenig Berücksichtigung finden. Erschwerend kommt laut den genannten Studien hinzu, dass Lernplattformen und -programme häufig keine Schnittstellen aufweisen und sich deshalb nicht oder nur schwer miteinander oder in schulische Datenbanken (z. B. zur Schülerinnen- und Schülerverwaltung, Stundenplangestaltung, digitales Klassenbuch) integrieren lassen. Somit stellen sie keine zusammenhängende Dokumentation für die Diagnose und Lernberatung bereit. Dieses Problem wird noch dadurch potenziert, dass, wie Bingham et al. (2018) feststellen, an den meisten Schulen nicht nur eine Plattform, sondern eine Fülle unterschiedlicher Programme, Webressourcen, Inhaltsanbieter und Geräte genutzt wird. Ein einzelnes Programm oder eine einzelne Plattform ist, laut der Erfahrung der Lehrkräfte, nicht in der Lage, die vielfältigen qualitativen Ansprüche zu erfüllen, die sie an das Lernmaterial für ihren Unterricht stellen. Die Arbeit mit unterschiedlichen Plattformen und Programmen erhöht jedoch den Aufwand, der für die technische Integration, die inhaltliche Abstimmung sowie die Einbindung in individuelle Lernpläne und schulinterne Curricula betrieben werden muss (vgl. auch Coiro et al. 2018; Yildirim et al. 2014).

\section{Anforderungen an räumliche Ressourcen}

Neben technischen werden in verschiedenen Studien auch neue Anforderungen an die räumlichen Ressourcen von Schulen angesprochen. Kallio (2018) dokumentiert anhand von vier Einzelfällen, wie Schulen ihre räumlichen Strukturen durch Umnutzung und Umbauten weiterentwickeln und neuen Bedürfnissen, die durch personalisiertes Lernen entstehen, anpassen. Existierende Schulgebäude mit ihren traditionellen Raumstrukturen erfordern dabei häufig Kompromisslösungen, da personalisiertes Lernen z. B. in grösserem Umfang Teilungsräume und individuelle Arbeitsplätze 
benötigt, als diese in traditionellen Schulgebäuden zur Verfügung stehen. Pane et al. (2017) stellen entsprechend fest, dass die räumlichen Gegebenheiten an den von ihnen untersuchten Schulen die Umsetzung personalisierten Lernens teilweise einschränken. Taylor (2016) weist darauf hin, dass auch innerhalb der Klassenräume eine flexible Möblierung, die sich je nach Bedarf arrangieren lässt, hilfreich ist.

\section{Rhythmisierung und Flexibilisierung}

Ein weiterer schulorganisatorischer Aspekt betrifft die Rhythmisierung des Schultags und, damit einhergehend, variierende Gruppierungen der Schülerinnen und Schüler entsprechend ihrer Lernbedürfnisse. Die Lehrkräfte an Projektschulen der Next Generation Learning Challenge in der Befragung von Pane et al. (2017) berichten insgesamt eine grössere Flexibilität in der Nutzung personeller, räumlicher und zeitlicher Ressourcen als Lehrpersonen, die nicht an Projektschulen unterrichten. Sie heben flexible Gruppierungen als Schlüsselstrategie zur Personalisierung des Lernens hervor. Allerdings stellt dieselbe Studie fest, dass die flexible Zusammensetzung von Lerngruppen vor allem innerhalb von Schulklassen erfolgt. Flexible Gruppierungen über Schulklassen oder gar Jahrgänge hinweg konnten dagegen nur an wenigen Schulen beobachtet werden. Einige Studien berichten über Veränderungen der Stundenpläne, etwa die Einführung von Unterrichtstagen mit Klassenunterricht im Wechsel mit Tagen, an denen die Schülerinnen und Schüler selbstgesteuert arbeiten (Bingham 2017) oder der Umstellung auf ein System mit Blockunterricht (Taylor 2016). Die Herausforderung besteht in schulorganisatorischer Hinsicht offenbar darin, ein System zu finden, dass flexibel genug die individuellen Lernbedürfnisse einzelner Schülerinnen und Schüler berücksichtigt, aber gleichzeitig strukturiert und übersichtlich ist und die Ressourcen der Schule nicht überfordert. So erwiesen sich in der Untersuchung von Pane et al. (2017) komplett individualisierte Stundenpläne, die wöchentlich auf der Grundlage der Lernfortschritte der Schülerinnen und Schüler aktualisiert wurden, als verwirrend für Schülerinnen und Schüler sowie aufgrund fehlender technischer, räumlicher und personeller Voraussetzungen als logistisch kaum umsetzbar.

Rolle der Schulleitungen

Schulleitungen spielen eine wichtige Rolle bei der Implementation digital gestützten personalisierten Lernens. Gross und DeArmond (2018) stellten fest, dass Schulleitungen sich, auch wenn sie die Einführung digital gestützten personalisierten Lernens generell befürworten, häufig damit schwertun, Ziele für die Implementierung digitalen personalisierten Lernens zu formulieren bzw. gemeinsame und geteilte Zielvorstellungen mit ihren Kollegien zu entwickeln. Einige Schulleitungen delegieren entsprechende Schulentwicklungsaufgaben an einzelne Lehrkräfte oder Lehrkräfteteams oder von aussen «eingekaufte» Coaches. Dies hat zur Folge, dass 
die Umsetzung personalisierten Lernens auf einzelne Lehrkräfte und Klassen beschränkt bleibt, die untereinander kaum abgestimmt sind und keinen gemeinsamen Prinzipien und Strukturen folgen. Auf diese Weise entwickeln sich allenfalls singuläre Best Practice-Beispiele, die keine Breitenwirkung entfalten können, was sowohl von den Lehrpersonen als auch von den Schülerinnen und Schülern als unbefriedigend empfunden wird. Erfolgreiche Schulleitungen zeichnen sich demgegenüber dadurch aus, dass sie, wie in den vorigen Abschnitten bereits dargestellt, Freiräume für die Erprobung unterschiedlicher Ansätze personalisierten Lernens schaffen, aber auch Teamstrukturen zur Erfahrungsweitergabe etablieren und die Erfahrungen schliesslich wieder zusammentragen, um schulweite Einigungen zu den Zielen, Verfahren und Standards personalisierten Lernens herbeizuführen (vgl. auch Basham et al. 2016; Bingham 2017; Myers 2018). Insgesamt scheint die Abstimmung gemeinsamer Ziele und Arbeitsformen nach Gross und DeArmond (2018) erleichtert zu werden, wenn die Schulen nach einem formalisierten Modell mit einer einheitlichen digitalen Lernplattform (wie etwa nach dem Summit-School-Modell) arbeiten, da dies bereits bestimmte Parameter personalisierten Lernens festlegt. In der Untersuchung von Bingham (2017) wurden darüber hinaus die Lernprozessdaten der Schülerinnen und Schüler genutzt, um mit den Lehrkräften die Qualität ihres Unterrichts zu reflektieren und sie in ihrer Verantwortung für die Lernfortschritte ihrer Schülerinnen und Schüler bzw. ihrer Rolle als Lernberatung zu bestärken (vgl. auch Myers 2018).

\section{Einbezug der Eltern}

Schliesslich werden die Akzeptanz und Unterstützung unterschiedliche externer Stakeholder-Gruppen problematisiert. Der Einbezug der Eltern erweist sich als bedeutsam, um Schülerinnen und Schüler beim personalisierten Lernen zu unterstützen und zu motivieren (Gallagher 2014; Myers 2018). Lehrpersonen nutzen verschiedene Strategien, um den Eltern Zugang zu den digitalen Lernsystemen zu ermöglichen und sie an der Überprüfung und Reflektion der Lernprozesse ihrer Kinder zu beteiligen. So führen sie Eltern in die Nutzung von Lernplattformen ein, schicken ihnen Lernstandsmitteilungen für ihre Kinder oder nutzen die systemgenerierten Daten für Elterngespräche (Amro und Borup 2019; Gallagher 2014; Yildirim et al. 2014). Auch Staub, Stebler und Reusser (2018) weisen auf die Notwendigkeit formalisierter Kommunikationswege, gerade an grösseren Schulen, hin und zeigen unterschiedliche Strategien auf, wie die Kooperation von Schule und Elternhaus von erfolgreichen Schulen gestaltet wird. Akzeptanzprobleme können sich dadurch einstellen, dass personalisiertes Lernen den eigenen Lernbiografien von Eltern zuwiderläuft (Myers 2018). 
Curriculare Vorgaben und zentrale Leistungsmessungen

Ein letzter problematischer Aspekt, der in mehreren Studien zur Sprache kommt, ist der Umgang mit übergeordneten curricularen Vorgaben sowie zentralisierten Leistungsmessungen und -standards. In der Untersuchung von Gross und DeArmond (2018) berichten Lehrkräfte wie auch Schülerinnen und Schüler über das Dilemma individualisierter Rückmeldungen und kriterialer, standardbasierter Leistungsbewertungen: Für Lehrkräfte ergibt sich ein Widerspruch daraus, individuelle Lernfortschritte an kriterialen Standards messen oder Lernprozesse zu personalisieren und gleichzeitig curriculare Vorgaben erfüllen zu sollen (Bingham 2017; Pane et al. 2017; Prain et al. 2013). Schülerinnen und Schüler bemängeln an einer Leistungsbewertung aufgrund individueller Lernfortschritte, angesichts bevorstehender Abschlussnoten und -zeugnisse nicht zu wissen, wo sie mit Blick auf übergreifende Standards stehen. Auch seitens der Schulverwaltungen wird die Anschlussfähigkeit an die herkömmliche Form der Leistungsbewertung, die für Übergänge zu anderen Schulen oder für den Schulabschluss notwendig ist, als Problem gesehen (Bingham 2017; Bingham et al. 2018; Gross und DeArmond 2018). So stehen Schulen, die an Pilotversuchen teilnehmen, vor dem Problem, dass für sie temporäre Ausnahmen von bestehenden Verwaltungsstrukturen und rechtlichen Rahmenbedingungen geschaffen, aber keine dauerhaften Lösungen gefunden wurden, was die Nachhaltigkeit ihrer Reformbemühungen unterminiert (Gross und DeArmond 2018).

\section{Schlussfolgerungen}

Bevor der Versuch einer Zusammenfassung unternommen wird, soll auf einige Begrenzungen dieser Analyse hingewiesen werden. Zunächst sind Untersuchungen aus dem US-amerikanischen Raum, wie bereits weiter oben erwähnt, in diesem Review überrepräsentiert. Die Ergebnisse spiegeln also zu einem gewissen Grad die Besonderheiten eines bestimmten Schulsystems wieder und lassen sich sicher nicht 1:1 auf den deutschsprachigen Raum übertragen. So ist für die vorliegende Frage nach der Implementation digital gestützten personalisierten Lernens von besonderer Bedeutung, dass computergestützter Unterricht in den USA eine wesentlich längere Tradition hat als im deutschsprachigen Raum. Es kann deshalb vermutet werden, dass bei der Implementierung digital gestützten personalisierten Lernens an Schulen in Deutschland grundsätzlichen Fragen von Ausstattung und technischen Handhabungskompetenzen in den Vordergrund drängen, die in den analysierten Studien keine Relevanz hatten.

Aber auch viele andere Rahmenbedingungen für die Entwicklung der Einzelschule weichen vom deutschsprachigen Kontext (der allerdings in dieser Hinsicht auch nicht homogen ist) ab, angefangen von Instrumenten der Schulsteuerung und Rechenschaftslegung bis hin zur Schulfinanzierung, Personalgewinnung sowie 
Aus- und Weiterbildung von Lehrkräften. Vor diesem Hintergrund wurde an die Synthese der Fülle von Einzelergebnissen ein spezifisch gefärbter Fokus herangetragen. Mit der Systematisierung anhand des Drei-Wege-Modells wurde der Blick speziell auf jene Felder und Entwicklungsbereiche gerichtet, die im deutschsprachigen Diskurs im Vordergrund stehen. Die Analyse setzt damit eigene Schwerpunkte und gewichtet die Ergebnisse einzelner Studien anders als die Studien selbst.

Die Analyse der empirischen Forschungsarbeiten vermittelt auf diese Weise trotz der genannten Einschränkungen ein facetten- und detailreiches Bild der Implementierung digital gestützten personalisierten Lernens, das auch für die hiesige inklusivmediale Schulentwicklung aufschlussreich ist. Die Ergebnisse untermauern in ihrer Vielfalt zunächst die eingangs getroffene Feststellung: Personalisiertes Lernen mit digitalen Medien wird nicht nur theoretisch sehr unterschiedlich verstanden, sondern weist auch in der Praxis ein weites Spektrum verschiedenartiger Umsetzungen auf. Damit zeigt sich in den empirischen Befunden ein Muster, das bereits aus der Untersuchung von inklusiver Schulentwicklung und Medienentwicklung an Schulen bekannt ist und auch für inklusiv-mediale Schulentwicklung gelten dürfte. In der schulpraktischen Implementation solcher Innovationen finden sich zahlreiche Abstufungen und Varianten - jede Schule findet am Ende eine ihren je eigene Rahmenbedingungen geschuldete Lösung, wie sie digital gestütztes personalisiertes Lernen umsetzt.

Analog zur Darstellung in Rolff und Thünken (2020) lassen sich dabei aber dennoch für die drei zentralen Felder der Schulentwicklung einzelfallübergreifende Aufgaben und Konfliktfelder identifizieren, die bei der Implementation digital gestützten personalisierten Lernens besonders im Vordergrund stehen. In Abbildung 3 werden diese nochmals stichpunktartig zusammengefasst: 


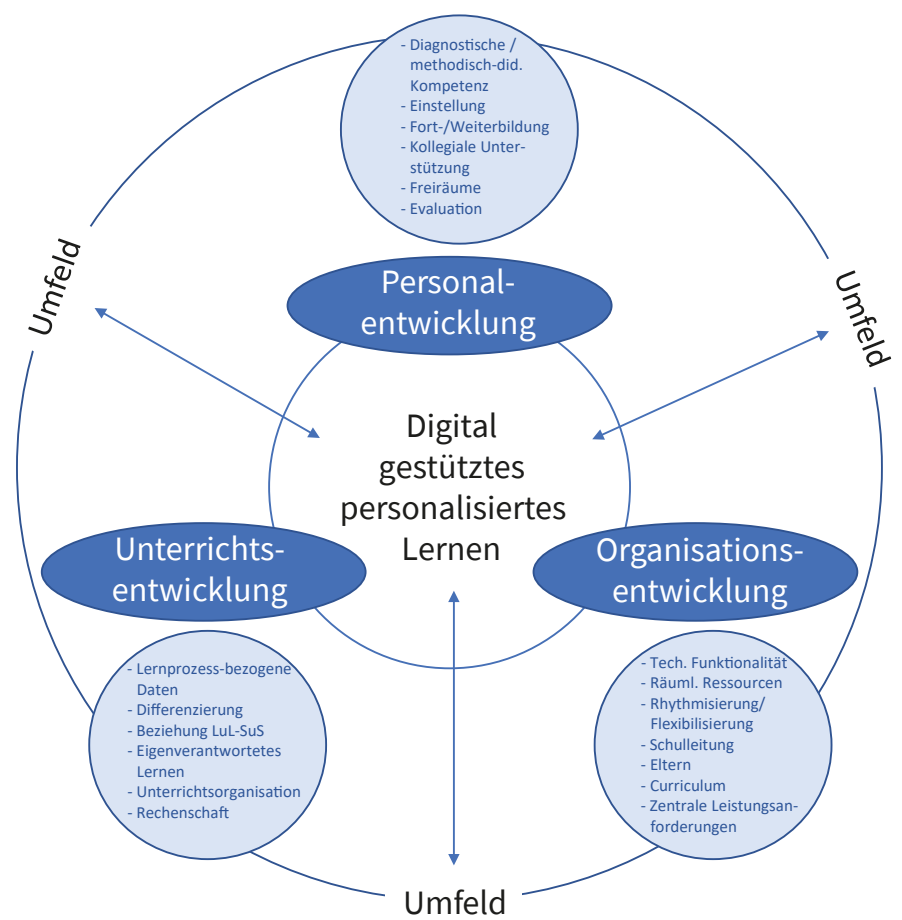

Abb. 3.: Zusammenfassende Darstellung der Entwicklungsfelder bei der Entwicklung des digital gestützten personalisierten Lernens.

Bei aller Unterschiedlichkeit zeigen die Evaluationen auch, dass einige Befürchtungen, die gelegentlich mit dem personalisierten Lernen und speziell mit dem Einsatz von auf künstlicher Intelligenz und Big Data basierenden digitalen Lernplattformen verbunden werden, unbegründet sind. So wird die Vermutung, dass digital gestütztes personalisiertes Lernen unweigerlich zu einer Isolierung der Lernenden führe (z. B. Spitzer 2016), durch die vorliegenden Ergebnisse nicht bestätigt. Vielmehr zeigt sich durchgängig das Bemühen um eine Balance von Individualisierung und dem Lernen in kleineren und grösseren Verbänden. Diese Beobachtung ist besonders wichtig mit Blick auf das Potenzial von digital gestütztem personalisierten Lernen für den inklusiven Unterricht, ist dessen Ziel doch ein gemeinsames Lernen aller Schülerinnen und Schüler. Auch für die inklusiv-mediale Schulentwicklung kann also geschlossen werden, dass ein verstärkter Einsatz individualisierter Lernprogramme wohl kaum zur Schule als «Lernfabrik» führen wird, in der die Schülerinnen und Schüler vereinzelt ihre individuellen computerisierten Lernpläne abarbeiten. Gleichzeitig belegen die Ergebnisse, dass das Finden eines ausgewogenen Gleichgewichts von Individualisierung und Gemeinschaft, von Offenheit und Struktur, sich als ein zähes Ringen erweist, und dies selbst wenn eine umfassend ausgebaute digitale Infrastruktur vorliegt und langjährige Erfahrung im Einsatz digitaler Lernmedien besteht. 
Auch zeigen die Ergebnisse, dass die Vorstellung, dass Lehrpersonen in naher Zukunft durch intelligente digitale Lernplattformen und -programme ersetzt werden (Ulbricht 2015), wohl kaum gerechtfertigt ist. Bislang erfüllen die eingesetzten Programme und Plattformen ganz offensichtlich nicht ansatzweise die komplexen Anforderungen, die eine individuelle Lernförderung in kognitiver, aber auch sozialer sowie emotional-motivationaler Hinsicht mit sich bringt, und es ist fraglich, ob sie dies je erfüllen können (und sollen). Vielmehr zeichnet sich ab, dass solche Programme Lehrkräfte unterstützen können, Schülerinnen und Schüler individuell im Unterricht zu fördern. Die Studien verweisen aber eindeutig auf die zentrale Rolle der Lehrkräfte, in deren Zuständigkeit die individuelle Auswahl des Lernmaterials genauso liegt wie die Bewertung von Lernfortschritten und die individualisierte Lernberatung. Die Personalisierung des Lernens liegt also, auch an Schulen, die mit digitalen Werkzeugen arbeiten, wesentlich in der Hand der Lehrkräfte. Der eigentliche Mehrwert digitaler Werkzeuge wird übereinstimmend in der Bereitstellung zusätzlicher Daten gesehen, auf die Lehrpersonen bei der Lernförderung zurückgreifen können und die vielfältig genutzt werden, um Lernprozesse zu reflektieren und Lernfortschritte transparent zu machen. Hierbei fällt - gerade mit Blick auf die Übertragbarkeit auf den deutschsprachigen Kontext - auf, dass in den vorliegenden Studien die Qualität der Daten, die digitale Lernplattformen liefern, kaum hinterfragt wird. Zwar werden mangelnde Integration, schlechte Verständlichkeit und fehlende Nutzerfreundlichkeit kritisiert, es erfolgt jedoch keine Diskussion der Programmstrukturen und Algorithmen, die hinter den Daten stehen. Die Validität der Daten als Indikatoren für Lernprozesse wird ebenso wenig infrage gestellt, wie Fragen des Datenschutzes und der Datenspeicherung thematisiert werden, die bei der Implementation an Schulen im deutschen Sprachraum immer mitbedacht werden müssen.

Wenig überraschend scheinen - jenseits der technischen Spezifika der eingesetzten digitalen Werkzeuge - in den Evaluationsergebnissen dieselben Faktoren auf, die grundsätzlich im Zusammenhang mit Schulentwicklung als Innovationsaufgabe diskutiert werden (Holtappels 2013; Rolff 1998): Auch bei der Implementierung digital gestützten personalisierten Lernens stellen sich etwa die Prozesssteuerung durch die Schulleitung, der Einbezug des gesamten Kollegiums, die Entwicklung geteilter Zielvorstellungen und schulweiter Absprachen und Regeln, die Etablierung von Kooperationsstrukturen und Erfahrungsweitergabe sowie das Angebot von Fortbildungen als wichtige Erfolgsbedingungen heraus. Es kann davon ausgegangen werden, dass sich gerade in diesem Bereich die Ergebnisse der vorliegenden Studien direkt auf die inklusiv-mediale Schulentwicklung übertragen lassen.

Konsequent zu Ende gedacht erweist sich digital gestütztes personalisiertes Lernen als eine Innovation, die so weitreichend ist, dass sie Einzelschulen an den Rand ihrer Handlungsspielräume bringt. Fast enttäuscht stellen einige der vorliegenden Evaluationen fest, dass sich bestehende schulische Strukturen, wie z. B. Schulklassen, 
Stundenpläne usw. nicht ohne Weiteres auflösen und in neue Strukturen überführen lassen, in der allein einzelne Lernende mit ihren je unterschiedlichen Lernbedürfnissen im Mittelpunkt stehen (Gross und DeArmond 2018; Pane et al. 2017). Die Evaluationen dokumentieren zahlreiche Barrieren auf unterschiedlichen Ebenen, die von etablierten Unterrichtspraxen und mangelnder Erfahrung über Kompetenzdefizite der Lehrkräfte bis zu fehlenden Ressourcen und mangelhaften organisatorischen Unterstützungsstrukturen sowie adversen ausserschulischen Rahmenbedingungen reichen. Mit Blick auf Perspektiven, die sich hieraus für die inklusiv-mediale Schulentwicklung ergeben, stellt sich an dieser Stelle die Frage, ob die Widerständigkeit schulischer Strukturen bei der Entwicklung neuer Konzepte nicht grössere Aufmerksamkeit erfahren sollte. Nimmt man die Befunde zur Veränderungsresistenz von Schule ernst, so ist auch infrage zu stellen, inwiefern die Forderung nach einer umfassenden Personalisierung von Lernprozessen vor dem Hintergrund schulpraktischer Erfahrungen realistisch ist. Für zukünftige Diskussionen um inklusiv-mediale Schulentwicklung regen sie dazu an, darüber nachzudenken, wieviel Personalisierung in der Praxis machbar und sinnvoll ist und wo die Grenzen der Personalisierung des Lernens liegen (sollten).

\section{Literatur}

Admiraal, Wilfried, Liesbeth Kester, Caressa Janssen, Mario de Jonge, Monika Louws, Lysanne Post, und Ditte Lockhorst. 2018. «Personalizing Learning with Mobile Technology in Secondary Education». In 14th International Conference Mobile Learning 2018. Proceedings of a meeting held 14-16 April 2018, Lisbon, Portugal. Bd. 1, herausgegeben von Inmaculada A. Sanchez, Pedro Isaias und Luis Rodrigues, 62-69. Red Hook, NY: Curran Associates, Inc. Zugriff am 10. August 2020. https://eric.ed.gov/?id=ED590358.

Amro, Falah, und Jered Borup. 2019. «Exploring Blended Teacher Roles and Obstacles to Success When Using Personalized Learning Software». Journal of Online Learning Research 5 (3): 229-50. Zugriff am 7. August 2020. https://www.proquest.com/docview/2396838263?a ccountid=11531.

Basham, James D., Tracey E. Hall, Richard A. Carter, und William M. Stahl. 2016. «An Operationalized Understanding of Personalized Learning». J Spec Educ Technol 31 (3): 126-36. https://doi.org/10.1177/0162643416660835.

Bingham, Andrea J. 2017. "Personalized learning in high technology charter schools». J Educ Change 18 (4): 521-49. https://doi.org/10.1007/s10833-017-9305-0.

Bingham, Andrea J., John F. Pane, Elizabeth D. Steiner, und Laura S. Hamilton. 2018. «Ahead of the Curve: Implementation Challenges in Personalized Learning School Models». Educational Policy 32 (3): 454-89. https://doi.org/10.1177/0895904816637688.

Boban, Ines, und Andreas Hinz. 2009. «Der Index für Inklusion». Sozial Extra 33 (9-10): 12-16.

Bosse, Ingo. 2019. «Digitalisierung und Inklusion». Schule inklusiv 4: 4-9. 
Coiro, Julie, Wendy Espinoza Cotta, Terry Deeney, Jay Fogleman, und Annice Correia Gabel. 2018. «Advancing A Shared Understanding of Personalized Learning: Insights from Eight Middle School Classrooms in Rhode Island». Unveröffentlichtes Manuskript. Zugriff am 7. August 2020. https://www.academia.edu/download/60262029/PL_Paper_20182019081117405-14tnjg7.pdf.

De Hond, Maurice, und Tijl Rood. 2017. «Flip the School, Forget the Classroom; How to Enable Personalised Learning with the Help of Information Technology». In Handbook on digital learning for K-12 schools, herausgegeben von Ann Marcus-Quinn und Tríona Hourigan, 31728. Switzerland: Springer. https://doi.org/10.1007/978-3-319-33808-8_18.

Dockterman, David. 2018. «Insights from 200+ years of personalized learning». npj Science of Learning 3 (1): 1-6. https://doi.org/10.1038/s41539-018-0033-x

Duckett, I. 2010. «Personalized Learning and Vocational Education and Training». In International encyclopedia of education, herausgegeben von Penelope L. Peterson, Eva L. Baker und Barry McGaw. 3. ed., 391-96. Oxford: Elsevier. https://doi.org/10.1016/B978-0-08-0448947.00789-2

Enyedy, Noel. 2014. «Personalized instruction: New interest, old rhetoric, limited results, and the need for a new direction for computer-mediated learning». Boulder, CO: National Education Policy Center. Zugriff am 21. August 2020. https://nepc.colorado.edu/publication/ personalized-instruction.

Feuser, Georg. 2013. «Die ,Kooperation am Gemeinsamen Gegenstand' - ein Entwicklung induzierendes Lernen». In Entwicklung und Lernen. Bd. 7, herausgegeben von Georg Feuser und Joachim Kutscher, 282-93. Stuttgart: Kohlhammer.

Filk, Christian. 2019a. «Onlife-Partizipation für alle! - Plädoyer für eine inklusiv-digitale Bildung». In Schule digital - wie geht das? herausgegeben von Olaf-Axel Burow, 62-82. Weinheim: Beltz-Verlag.

Gallagher, Rodney P. 2014. «Implementations of technology enhanced personalized learning: Explorations of success criteria, concerns, and characteristics». Dissertation, Graduate School of Education and Psychology, Pepperdine University. Zugriff am 20. August 2020. https://digitalcommons.pepperdine.edu/etd/448.

Galle, Marco, Rita Stebler, und Kurt Reusser. 2019. «Vom Einzelkämpfer zum Teamplayer: Kokonstruktive Kooperation in Grundschulen mit personalisierten Lernkonzepten». In Grundschulpädagogik zwischen Wissenschaft und Transfer, herausgegeben von Christian Donie, Frank Foerster, Marlene Obermayr, Anne Deckwerth, Gisela Kammermeyer, Gerlinde Lenske, Miriam Leuchter und Anja Wildemann, 194-99. Wiesbaden: VS Verlag für Sozialwissenschaften. https://doi.org/10.1007/978-3-658-26231-0_24.

Graf, Sabine, und Kinshuk. 2012. «Personalized Learning». In Encyclopedia of the Sciences of Learning, herausgegeben von Norbert Seel, 2592-93. Boston: Springer. https://doi. org/10.1007/978-1-4419-1428-6_151.

Gross, Betheny, und Michael DeArmond. 2018. «Personalized Learning at a Crossroads: Early Lessons from the Next Generation Systems Initiative and the Regional Funds for Breakthrough Schools Initiative». Unveröffentlichtes Manuskript. 
Hartung, Julia, Elsa Zschoch, und Michael Wahl. 2021. «Inklusion Und Digitalisierung in Der Schule: Gelingensbedingungen Aus Der Perspektive Von Lehrerinnen Und Lehrern Sowie Schülerinnen Und Schülern». MedienPädagogik: Zeitschrift für Theorie Und Praxis Der Medienbildung 41 (Inklusiv-mediale Bildung):55-76. https://doi.org/10.21240/ mpaed/41/2021.02.04.X.

Holmes, Wayne, Stamatina Anastopoulou, Heike Schaumburg, und Manolis Mavrikis. 2018. Technology-enhanced personalised learning: Untangling the evidence. Stuttgart: Robert Bosch Stiftung $\mathrm{GmbH}$.

Holtappels, Heinz Günter. 2013. «Schulentwicklung und Lehrerkooperation». In Empirische Bildungsforschung. Theorien, Methoden, Befunde und Perspektiven herausgegeben von Nele McElvany und Heinz Günther Holtappels, 35-62. Münster: Waxmann.

Kallick, Bena, und Allison Zmuda. 2017. Students at the center: Personalized learning with habits of mind. Alexandria, VA: ASCD.

Kallio, Julie M. 2018. «Participatory Design of Classrooms: Infrastructuring Education Reform in K-12 Personalized Learning Programs». Journal of Learning Spaces 7 (2): 35-49. Zugriff am 7. August 2020. https://www.proquest.com/docview/2228638448?accountid=11531.

Lee, Dabae, Yeol Huh, Chun-Yi Lin, und Charles M. Reigeluth. 2018. «Technology functions for personalized learning in learner-centered schools». Education Tech Research Dev 66 (5): 1269-1302. https://doi.org/10.1007/s11423-018-9615-9.

Myers, Rebecca G. 2018. «Transitioning to a Personalized Learning Environment Leveraging One-to-One Devices». Dissertation, Bagwell College of Education, Kennesaw State University. Zugriff am 17. August 2020. https://digitalcommons.kennesaw.edu/educleaddoc_ etd $/ 14$.

Netcoh, Steven, und Penny A. Bishop. 2017. «Personalized Learning in the Middle Grades: A Case Study of One Team's Successes and Failures». Middle Grades Research Journal 11 (2): 33-48.

Pane, John F., Elizabeth D. Steiner, Mathew D. Baird, Laura S. Hamilton, und Joseph D. Pane. 2017. «Informing Progress: Insights on Personalized Learning Implementation and Effects». Unveröffentlichtes Manuskript. Zugriff am 7. August 2020. https://pdfs.semanticscholar. org/d586/d547731fbd5811420dda369e26ecec76decd.pdf.

Prain, Vaughan, Peter Cox, Craig Deed, Jeffrey Dorman, Debra Edwards, Cathleen Farrelly, Mary Keeffe et al. 2013. «Personalised learning: lessons to be learnt». British Educational Research Journal 39 (4): 654-76. https://doi.org/10.1080/01411926.2012.669747.

Reusser, Kurt, Christine Pauli, und Rita Stebler. 2018. "Personalisiertes Lernen: zur Analyse eines Bildungsschlagwortes und erste Ergebnisse aus der perLen-Studie». Zeitschrift für Pädagogik 64 (2): 159-78.

Rolff, Hans-Günter. 1998. «Entwicklung von Einzelschulen: viel Praxis, wenig Theorie und kaum Forschung - ein Versuch, Schulentwicklung zu systematisieren». In Jahrbuch der Schulentwicklung 1, herausgegeben von Hans-Günter Rolff, Georg Hansen, Klaus Klemm und KlausJürgen Tillmann, 295-326. Weinheim, Basel: Beltz. 
Rolff, Hans-Günter, und Ulrich Thünken. 2020. Digital gestütztes Lernen: Praxisbeispiele für eine zeitgemäße Schulentwicklung. Weinheim, Basel: Beltz.

Schaumburg, Heike. 2021. «Personalisierung mit digitalen Medien». In Kompetenzorientiert Unterrichten und Lernen mit digitalen Medien, herausgegeben von Hans-Günther Rolff und Arnold Brägger 382-399. Weinheim, Basel: Beltz.

Schaumburg, Heike. 2020. «Inklusion durch Personalisierung? Potenziale personalisierter Lernumgebungen für einen inklusiven Unterricht». Computer + Unterricht (117): 9-13.

Schaumburg, Heike, und Doreen Prasse. 2019. Medien und Schule. Bad Heilbrunn: Klinkhardt utb.

Schratz, Michael, und Tanja Westfall-Greiter. 2010. «Das Dilemma der Individualisierungsdidaktik. Plädoyer für personalisiertes Lernen in der Schule». Journal für Schulentwicklung 1 (2010): 18-31.

Schulz, Lea, Igor Krstoski, Nils Lion, und Dirk Neumann. 2019. «Digital-inklusiver Unterricht: Didaktische Integration digitaler Medien im gemeinsamen Unterricht». Schule inklusiv (4): $10-15$.

Schulz, Lea. 2021. «Diklusive Schulentwicklung: Erfahrungen Und Erkenntnisse Der DigitalInklusiven Multiplikatorinnen- Und Multiplikatorenausbildung in Schleswig-Holstein». MedienPädagogik: Zeitschrift für Theorie Und Praxis Der Medienbildung 41 (Inklusiv-mediale Bildung):32-54. https://doi.org/10.21240/mpaed/41/2021.02.03.X.

Schulz-Zander, Renate. 1999. «Neue Medien und Schulentwicklung». In Schulentwicklung und Schulqualität. Beiträge zur Bildungsforschung und Schulentwicklung 8, herausgegeben von Ernst Rösner, 35-56. Dortmund: IFS-Verlag.

Spitzer, Manfred. 2016. «Risiken und Nebenwirkungen digitaler Informationstechnik: Anhörung durch die Enquetekommission ,Kein Kind zurücklassen-Rahmenbedingungen, Chancen und Zukunft schulischer Bildung in Hessen', Thema ,Digitalisierung' im Hessischen Landtag am 14.10. 2016». Unveröffentlichtes Manuskript. Zugriff am 7. August 2020. http:// www.aufwach-s-en.de/wp-content/uploads/2017/06/Spitzer_Hessischer_Landtag_2016. pdf.

Staub, Katriina Vasarik, Rita Stebler, und Kurt Reusser. 2018. «'In Parents' School Experience, the Teacher was just Lecturing at the Front'. School-Family Partnerships in Schools with Personalized Learning Concepts». International Journal about Parents in Education 10 (1): $1-13$.

Taylor, Bridget E. 2016. «A Case Study of Middle School Teachers' Experiences with Personalized Learning». Dissertation, Tift College of Education, Mercer University. Zugriff am 7. August 2020. https://search.proquest.com/docview/1826827728?accountid=11531.

Thomas, James, und Angela Harden. 2008. «Methods for the Thematic Synthesis of Qualitative Research in Systematic Reviews». BMC medical research methodology 8 (45). https://doi. org/10.1186/1471-2288-8-45.

Tong, Allison, Kate Flemming, Elizabeth McInnes, Sandy Oliver, und Jonathan Craig. 2012. «Enhancing Transparency in Reporting the Synthesis of Qualitative Research: ENTREQ». BMC medical research methodology 12 (181): 1-8. https://doi.org/10.1186/1471-2288-12-181. 
Ulbricht, Arne. 2015. Schule ohne Lehrer? Zurück in die Zukunft. 1. Auflage. EBL-Schweitzer. Göttingen: Vandenhoeck \& Ruprecht.

Yildirim, Zahide, Charles M. Reigeluth, Seolim Kwon, Yuichi Kageto, und Zihang Shao. 2014. «A comparison of learning management systems in a school district: searching for the ideal personalized integrated educational system (PIES)». Interactive Learning Environments 22 (6): 721-36. https://doi.org/10.1080/10494820.2012.745423.

Zylka, Johannes. 2018. Digitale Schulentwicklung: das Praxisbuch für Schulleitung und Steuergruppen. Weinheim: Beltz. 